\title{
GEOMETRIC PROPERTIES OF BANACH SPACES AND THE EXISTENCE OF NEAREST AND FARTHEST POINTS
}

STEFAN COBZAŞ

Received 19 November 2003

The aim of this paper is to present some generic existence results for nearest and farthest points in connection with some geometric properties of Banach spaces.

\section{Introduction}

The aim of this paper is to present some existence results for nearest-point and farthestpoint problems, in connection with some geometric properties of Banach spaces. The idea goes back to Efimov and Stečkin who, in a series of papers (see [28, 29, 30, 31]), realized for the first time that some geometric properties of Banach spaces, such as strict convexity, uniform convexity, reflexivity, and the Kadec-Klee property, and so forth, characterize some generic results concerning the uniqueness and existence of nearest points.

Let $X$ be a real normed linear space and $Z$ a nonempty subset of $X$. For $x \in X$, put

$$
\begin{gathered}
d(x, Z)=\inf \{\|x-z\|: z \in Z\}, \\
P(x)=P_{Z}(x)=\{z \in Z:\|x-z\|=d(x, Z)\}, \\
E(Z)=\{x \in X: P(x) \neq \varnothing\} \\
U(Z)=\{x \in X: \operatorname{card} P(x) \leq 1\} \\
E U(Z)=\{x \in X: \operatorname{card} P(x)=1\}
\end{gathered}
$$

The elements (if any) of the set $P_{Z}(x)$ are called nearest points to $x$ in $Z$. The set $Z$ is called proximinal if $E(Z)=X$, antiproximinal if $E(Z)=Z$, a uniqueness set if $U(Z)=X$, and Chebyshev if $E U(Z)=X$. If these sets only contain a subset which is $G_{\delta}$ and dense in $X$ (or, equivalently, if their complements are of the first Baire category in $X$ ), then the set $Z$ is called almost proximinal, an almost uniqueness set, almost Chebyshev, respectively. 
Suppose further that the set $Z$ is bounded and let

$$
\begin{gathered}
q(x, Z)=\sup \{\|x-z\|: z \in Z\}, \\
Q(x)=Q_{Z}(x)=\{z \in Z:\|x-z\|=h(x, Z)\}, \\
e(Z)=\{x \in X: Q(x) \neq \varnothing\}, \\
u(Z)=\{x \in X: \operatorname{card} Q(x) \leq 1\}, \\
e u(Z)=\{x \in X: \operatorname{card} Q(x)=1\} .
\end{gathered}
$$

The elements of the (possibly empty) set $Q_{Z}(x)$ are called farthest points from $x$ in $Z$. The set $Z$ is called remotal if $Q_{Z}(x) \neq \varnothing$ for every $x \in X$, and uniquely remotal if card $Q_{Z}(x)=$ 1 for every $x \in X$. As in the case of nearest points, the terms almost remotal and almost uniquely remotal are used to designate the fact that the set $X \backslash e(Z)$, (resp., $X \backslash e u(Z))$ is of the first Baire category.

In the following proposition, we collect some simple but useful properties of the distance function and of the projection.

Proposition 1.1. Let $Z$ be a nonempty set of a normed space $X$, and $x$, y elements of $X$. Then

(1) $d(x, Z)=d(x, \bar{Z})$ and $d(x, Z)=0 \Leftrightarrow x \in \bar{Z}$;

(2) the function $d(\cdot, Z)$ is nonexpansive, that is,

$$
\forall x_{1}, x_{2} \in X, \quad\left|d\left(x_{1}, Z\right)-d\left(x_{2}, Z\right)\right| \leq\left\|x_{1}-x_{2}\right\|,
$$

hence uniformly continuous on $X$;

(3) $d(x+y, y+Z)=d(x, Z)$ and $P_{y+Z}(x+y)=P_{Z}(x), d(x, \alpha Z)=|\alpha| d\left(\alpha^{-1} x, Z\right)$, and $P_{\alpha Z}(x)=P_{Z}\left(\alpha^{-1} x\right)$, for $\alpha \neq 0$.

For the convenience of the reader, we will recall some geometric properties of normed spaces. If $X$ is a normed space, then, for $x \in X$ and $r>0$, denote by $B(x, r)=\{y \in X$ : $\|y-x\| \leq r\}$ (resp., $\left.B^{\prime}(x, r)=\{y \in X:\|y-x\|<r\}\right)$ the closed (resp., open) balls in $X$, and let $S(x, r)=\{y \in X:\|y-x\|=r\}$ be the sphere of center $x$ and radius $r$. Let also $B_{X}=B(x, 1), B_{X}^{\prime}=B^{\prime}(o, 1)$, and $S_{X}=S(0,1)$ be the closed unit ball, the open unit ball, and the unit sphere of $X$, respectively.

For $x, y \in X$, denote by $[x ; y]=\{x+t(y-x): t \in[0 ; 1]\}$ the closed interval joining $x$ and $y$. The intervals $[x ; y),(x ; y]$, and $(x ; y)$ are defined in an obvious way. The ray $R(x, y)$ is defined by $R(x, y)=\{x+t(y-x): t \in[0 ; \infty)\}$ and the line passing through $x$ and $y$ by $D(x, y)=\{x+t(y-x): t \in \mathbb{R}\}$.

Strict convexity. The normed space $X$ is called strictly convex (or rotund) if its unit sphere $S_{X}$ does not contain nontrivial segments, that is, for every $x, y \in S_{X}, x \neq y$, and $t \in(0 ; 1)$, we have $\|(1-t) x+t y\|<1$, or, equivalently, if the equality $\|(1-t) x+t y\|=1$ holds for some $x, y \in S_{X}$ and some $t \in(0 ; 1)$, then $x=y$.

The following proposition contains some equivalent conditions for strict convexity. 
Proposition 1.2. For a normed space $X$, the following conditions are equivalent:

(1) $X$ is strictly convex;

(2) for very $x, y \in S_{X}$ with $x \neq y,\|x+y\|<2$;

(3) for every $x, y \in X \backslash\{0\}$, the equality $\|x+y\|=\|x\|+\|y\|$ implies $y=\alpha x$ for some $\alpha>0$.

Proof. Condition (2) follows from (1) taking $t=1 / 2$ in the definition of strict convexity. The implication $(2) \Rightarrow(1)$ follows from the convexity of the function $\varphi(t)=\|x+t(y-x)\|$, $t \in[0,1]$.

(1) $\Rightarrow(3)$ Suppose that the equality $\|x+y\|=\|x\|+\|y\|$ holds for some nonzero $x, y \in$ $X$. It can be written in the equivalent form

$$
\left\|\frac{\|x\|}{\|x\|+\|y\|} \cdot \frac{x}{\|x\|}+\frac{\|y\|}{\|x\|+\|y\|} \cdot \frac{y}{\|y\|}\right\|=1,
$$

which, by the strict convexity of $X$, implies $y=\alpha x$ with $\alpha=\|y\| /\|x\|$.

(3) $\Rightarrow$ (2) If $\|x+y\|=2=\|x\|+\|y\|$ for some $x, y \in S_{X}$, then $y=\alpha x$ for some $\alpha>0$. Since $\|y\|=\|x\|=1$, it follows $\alpha=1$ and $y=x$.

Uniform convexity. A normed space $X$ is called uniformly convex (or uniformly rotund) if for every $\epsilon, 0<\epsilon \leq 2$, there exists $\delta=\delta(\epsilon)>0$ such that for every $x, y \in B_{X}$,

$$
\left\|\frac{x+y}{2}\right\|>1-\delta \Longrightarrow\|x-y\|<\epsilon
$$

or, equivalently,

$$
\|x-y\| \geq \epsilon \Longrightarrow\left\|\frac{x+y}{2}\right\| \leq 1-\delta
$$

The modulus of convexity of a normed space $X$ is the function $\delta_{X}:[0 ; 2] \rightarrow[0 ; 1]$, defined by one of the following equivalent conditions:

$$
\begin{aligned}
\delta_{X}(\epsilon) & =\inf \left\{1-\left\|\frac{x+y}{2}\right\|: x, y \in S_{X},\|x-y\| \geq \epsilon\right\} \\
& =\inf \left\{1-\left\|\frac{x+y}{2}\right\|: x, y \in B_{X},\|x-y\| \geq \epsilon\right\} .
\end{aligned}
$$

For $0 \leq \epsilon<2$, equalities of the above type hold also with the condition $\|x-y\|>\epsilon$. see, for instance, [58, pages 442-446].

Obviously the space $X$ is uniformly convex if and only if $\delta_{X}(\epsilon)>0$ for every $\epsilon, 0<\epsilon \leq 2$.

The modulus of convexity satisfies $\delta_{X}(\epsilon) \leq \epsilon / 2$ and $\delta_{X}(\epsilon) \leq \delta_{X}\left(\epsilon^{\prime}\right)$ if $0 \leq \epsilon \leq \epsilon^{\prime}$. If $X$ is uniformly convex, then the mutually equivalent conditions (1.5) and (1.6) hold with $\delta=\delta_{X}(\epsilon)$. 
By the monotonicity of the modulus $\delta_{X}$, conditions (1.5) and (1.6) are also equivalent with

$$
\|x+y\| \leq 2\left(1-\delta_{X}(\|x-y\|)\right)
$$

for all $x, y \in B_{X}, x \neq y$.

Uniform convexity can be characterized in terms of some sequences in the unit ball of $X$.

Proposition 1.3 [58, page 447]. For a normed space X, the following conditions are equivalent:

(1) $X$ is uniformly convex;

(2) if $\left(x_{n}\right)$ and $\left(y_{n}\right)$ are two sequences in the unit sphere $S_{X}$ of $X$ such that $\left\|x_{n}+y_{n}\right\| \rightarrow 2$ for $n \rightarrow \infty$, then $\left\|x_{n}-y_{n}\right\| \rightarrow 0$;

(3) if $\left(x_{n}\right)$ and $\left(y_{n}\right)$ are two sequences in the unit ball $B_{X}$ of $X$ such that $\left\|x_{n}+y_{n}\right\| \rightarrow 2$ for $n \rightarrow \infty$, then $\left\|x_{n}-y_{n}\right\| \rightarrow 0$;

(4) if $\left(x_{n}\right)$ and $\left(y_{n}\right)$ are two sequences in $X$ such that $\left(\left\|x_{n}\right\|\right),\left(\left\|y_{n}\right\|\right),\left(\left\|2^{-1}\left(x_{n}+y_{n}\right)\right\|\right)$ all tend to some $d>0$ for $n \rightarrow \infty$, then $\left\|x_{n}-y_{n}\right\| \rightarrow 0$.

Local uniform convexity. If for fixed $x \in S_{X}$ and every $\epsilon, 0<\epsilon \leq 2$, there exists $\delta=\delta(\epsilon$, $x)>0$ such that one of the equivalent conditions (1.5) or (1.6) holds, then the space $X$ is called locally uniformly convex (or locally uniformly rotund). Obviously uniform convexity implies local uniform convexity and, in its turn, this implies strict convexity. A modulus of local uniform convexity can be defined as a localized version of the modulus of uniform convexity. For $x \in S_{X}$ and $0<\epsilon \leq 2$, put

$$
\begin{aligned}
\delta_{X}(\epsilon, x) & =\inf \left\{y \in S_{X}:\|x-y\| \geq \epsilon\right\} \\
& =\inf \left\{y \in B_{X}:\|x-y\| \geq \epsilon\right\} .
\end{aligned}
$$

Obviously the space $X$ is locally uniformly convex if and only if $\delta_{X}(\epsilon, x)>0$ for every $x \in$ $S_{X}$ and every $\epsilon, 0<\epsilon \leq 2$. In this case, conditions (1.5) and (1.6) hold with $\delta=\delta_{X}(\epsilon, x)$. Condition (1.8) holds also with $\delta_{X}(\|x-y\|, x), x \in S_{x}, y \in B_{X}, x \neq y$, instead of $\delta_{X}(\| x-$ $y \|)$.

A theorem similar to Proposition 1.3 but localized, that is, with $x_{n}=x \in S_{X}$, holds for local uniform convexity too.

Since the unit ball of a normed space $X$ determines the shape of all balls in $X$, the conditions of uniform, and of local uniform convexity, can be written for arbitrary balls.

Let $B\left(x_{0}, r\right)$ be an arbitrary closed ball in a normed space $X$.

Proposition 1.4. (1) Suppose that the space $X$ is uniformly convex with modulus of uniform convexity $\delta_{X}$. Then for every $z, z^{\prime} \in B\left(x_{0}, r\right), z \neq z^{\prime}$, the following inequality holds:

$$
\left\|z+z^{\prime}-2 x_{0}\right\| \leq 2 r(1-\delta),
$$

where

$$
\delta=\delta_{X}\left(\frac{\left\|z-z^{\prime}\right\|}{r}\right) .
$$


If $z, z^{\prime} \in B\left(x_{0}, r\right)$ satisfy $\left\|z-z^{\prime}\right\| \geq \epsilon$ for some $\epsilon \in(0 ; 2]$, then inequality (1.10) holds with $\delta=\delta_{X}(\epsilon / r)$.

(2) If $X$ is locally uniformly convex with modulus of local uniform convexity $\delta_{X}(\cdot, \cdot)$ and $z \in S\left(x_{0}, r\right), z^{\prime} \in B\left(x_{0}, r\right), z \neq z^{\prime}$, then inequality (1.10) holds with

$$
\delta=\delta_{X}\left(\frac{\left\|z-z^{\prime}\right\|}{r}, \frac{z-x_{0}}{r}\right)
$$

If $z \in S\left(x_{0}, r\right)$ and $z^{\prime} \in B\left(x_{0}, r\right)$ are such that $\left\|z-z^{\prime}\right\| \geq \epsilon$, for some $\epsilon \in(0 ; 2]$, then (1.10) holds with

$$
\delta=\delta_{X}\left(\frac{\epsilon}{r}, \frac{z-x_{0}}{r}\right)
$$

Proof. Applying (1.8) to $\left(z-x_{0}\right) / r$ and $\left(z^{\prime}-x_{0}\right) / r$, one obtains

$$
\left\|z+z^{\prime}-2 x_{0}\right\| \leq 2 r(1-\delta)
$$

with $\delta=\delta_{X}\left(\left\|z-z^{\prime}\right\| / r\right)$ if $X$ is uniformly convex, (resp., $\delta=\delta_{X}\left(\left\|z-z^{\prime}\right\| / r,\left(z-x_{0}\right) / r\right.$ ) if $X$ is locally uniformly convex).

The assertions involving $\epsilon$ follow from the monotonicity of the moduli of uniform and of local uniform convexity.

A normed space $X$ is said to have the Kadec-Klee property provided $\left(x_{n}\right)$ tends weakly to $x$ and $\left\|x_{n}\right\| \rightarrow\|x\|$ implies $\left\|x_{n}-x\right\| \rightarrow 0$, that is, $\left(x_{n}\right)$ tends strongly to $x$, for every sequence $\left(x_{n}\right)$ in $X$ and $x \in X$. The space $\ell^{1}(\Gamma)$ and any locally uniformly convex space have the Kadec-Klee property. The Kadec-Klee property is called in [58] the RadonRiesz property, motivated by the fact that J. Radon and F. Riesz proved that the space $L^{p}(\Omega, \mathcal{A}, \mu)$ has this property for $1<p<\infty$.

\section{The problem of nearest points}

The geometric properties we considered at the end of the preceding section are closely related to the approximation properties of the space $X$. First we will examine strict convexity.

Proposition 2.1. For a normed space $X$, the following conditions are equivalent:

(1) the space $X$ is strictly convex;

(2) every nonempty convex subset of $X$ is a uniqueness set for best approximation;

(3) every nonempty closed convex subset of $X$ is a uniqueness set for best approximation.

Proof. (1) $\Rightarrow(2)$ Let $Z$ be a nonempty convex subset of $X$ and suppose that for some $x \in X$, there are $z_{1}, z_{2} \in Z$ such that $\left\|x-z_{1}\right\|=\left\|x-z_{2}\right\|=d(x, Z)=d>0$. Then $\left(z_{1}+z_{2}\right) / 2 \in Z$ and

$$
d \leq\left\|x-\frac{z_{1}+z_{2}}{2}\right\|=\frac{1}{2}\left(\left\|x-z_{1}+x-z_{2}\right\|\right) \leq \frac{1}{2}\left(\left\|x-z_{1}\right\|+\left\|x-z_{2}\right\|\right)=d
$$


implying $\left\|x-z_{1}+x-z_{2}\right\|=2 d=\left\|x-z_{1}\right\|+\left\|x-z_{2}\right\|$. By the strict convexity of $X$, there is $\alpha>0$ such that $x-z_{2}=\alpha\left(x-z_{1}\right)$. Because $\left\|x-z_{1}\right\|=\left\|x-z_{2}\right\|=d$, it follows that $\alpha=1$ and $z_{1}=z_{2}$.

The implication $(2) \Rightarrow(3)$ is obvious. To prove $(3) \Rightarrow(1)$ suppose that $X$ is not strictly convex. Then there exist $x_{0}, x_{1} \in S_{X}, x_{0} \neq x_{1}$, such that $\left\|x_{0}+x_{1}\right\|=2$. The convexity of the function $\varphi(t)=\left\|x_{0}+t\left(x_{1}-x_{0}\right)\right\|, t \in[0 ; 1]$, implies $\|0-\varphi(t)\|=1=d\left(0,\left[x_{0} ; x_{1}\right]\right)$ for all $t \in[0 ; 1]$.

In uniformly convex spaces, stronger results on the existence and uniqueness of best approximation hold.

Proposition 2.2. If $Z$ is a nonempty complete convex subset of a uniformly convex normed space $X$, then $Z$ is a Chebyshev set in $X$.

For the proof, we need a lemma.

Lemma 2.3. Let $X$ be a uniformly convex normed space. If $\left(x_{n}\right)$ is a sequence in $X$ such that, for some $d>0,\left\|x_{n}\right\| \rightarrow d$ for $n \rightarrow \infty$, and $\left\|x_{n}+x_{m}\right\| \rightarrow 2 d$, for $n, m \rightarrow \infty$, then the sequence $\left(x_{n}\right)$ is fundamental.

Proof. For $\epsilon>0$, let $\delta>0$ be such that (1.5) holds. Take $\alpha \in \mathbb{R}$ satisfying the conditions:

$$
0<\alpha<1, \quad \alpha<d, \quad \frac{2 \alpha}{d+\alpha}<\delta
$$

By hypothesis, there exists $n_{0} \in \mathbb{N}$ such that

$$
\forall n \geq n_{0}, \quad\left\|x_{n}\right\| \leq d+\alpha, \quad \forall n, m \geq n_{0}, \quad\left\|\frac{x_{n}+x_{m}}{2}\right\| \geq d-\alpha .
$$

Then $x_{n}^{\prime}=(d+\alpha)^{-1} x_{n} \in B_{X}$ and

$$
\left\|\frac{x_{n}^{\prime}+x_{m}^{\prime}}{2}\right\|=\frac{1}{d+\alpha}\left\|\frac{x_{n}+x_{m}}{2}\right\| \geq \frac{d-\alpha}{d+\alpha}=1-\frac{2 \alpha}{d+\alpha}>1-\delta
$$

for all $n, m \geq n_{0}$. It follows that $\left\|x_{n}^{\prime}-x_{m}^{\prime}\right\|<\epsilon$, so that $\left\|x_{n}-x_{m}\right\|<\epsilon(d+\alpha)<\epsilon(d+1)$ for all $n, m \geq n_{0}$. The lemma is proved.

Proof of Proposition 2.2. For $x \in X \backslash Z$, let $d=d(x, Z)>0$ and let $\left(z_{n}\right)$ be a minimizing sequence in $Z$, that is, $\left\|x-z_{n}\right\| \rightarrow d$. Reasoning as in the proof of Proposition 2.1, it follows

$$
\left\|\frac{x-z_{n}+x-z_{m}}{2}\right\|=\left\|x-\frac{z_{n}+z_{m}}{2}\right\| \rightarrow d
$$

By the above lemma, the sequence $\left(x-z_{n}\right)$ is fundamental. But then $\left(z_{n}\right)$ is also a fundamental sequence and, by the completeness of the set $Z$, it has a limit $z \in Z$. It follows $\|x-z\|=\lim _{n}\left\|x-z_{n}\right\|=d(x \cdot Z)$. Since every uniformly convex space is strictly convex, the uniqueness of $z$ follows from Proposition 2.1.

Proposition 2.2 is proved. 
Stečkin's results on best approximation by elements of arbitrary sets. In the seminal paper [76], Stečkin proved some fundamental generic uniqueness and existence results for nearest points in arbitrary subsets of normed spaces, and posed several open questions. This drew the attention of mathematicians interested in abstract approximation theory or in Banach space geometry, to this challenging area of research, leading to a series of papers dealing with generic existence and uniqueness for the nearest-point and farthest-point problems in normed spaces.

Now, we will present Stečkin's results along with the problems raised by him in [76]. As Stečkin's paper is available only in Russian, I will present the proofs, taking into account some subsequent developments, mainly by De Blasi, Myjak, and Papini [17, 19]. (An English translation of Stečkin's paper can be obtained upon request from the author of the present paper.) A subset $Z$ of a normed space $X$ is called relatively boundedly compact if its intersection with any closed ball in $X$ is relatively compact or, equivalently, if any bounded sequence in $Z$ contains a convergent subsequence. If the intersection of $Z$ with any closed ball is compact, then we call it boundedly compact.

We first present the results concerning uniqueness.

Theorem 2.4. Let $X$ be strictly convex normed space. Then

(1) the set $U(Z)$ is dense in $X$ for any $Z \subset X$,

(2) if $X$ is Banach and $Z$ is relatively boundedly compact, then $X \backslash U(Z)$ is of the first Baire category,

(3) if $X$ is Banach and $Z$ is closed and relatively boundedly compact, then $X \backslash U(Z)$ is of the first Baire category and $a G_{\delta}$ set.

The proof is based on the following simple remark.

Lemma 2.5. Let $X$ be a strictly convex normed space, $Z$ a nonempty subset of $X$, and $x_{0} \in$ $X \backslash Z$.

If $z_{0} \in P_{Z}\left(x_{0}\right)$, then $P_{Z}(x)=\left\{z_{0}\right\}$ for every $x \in\left[z_{0} ; x_{0}\right)$.

Proof. The assertion is obviously true for $x=z_{0}$. Let $x \in\left(z_{0} ; x_{0}\right)$ and suppose that there exists $z \in Z \backslash\left\{z_{0}\right\}$ such that $\|x-z\| \leq\left\|x-z_{0}\right\|$. If $z \in\left(z_{0} ; x\right)$, then $\|x-z\|<\left\|x-z_{0}\right\|$, a contradiction. If $z$ does not lie on the line $D\left(z_{0}, x_{0}\right)$, then, by the strict convexity of $X$, we obtain again a contradiction:

$$
\left\|x_{0}-z\right\|<\left\|x_{0}-x\right\|+\|x-z\| \leq\left\|x_{0}-x\right\|+\left\|x-z_{0}\right\|=\left\|x-z_{0}\right\| .
$$

\section{Proof of Theorem 2.4.}

Proof of assertion (1). If $x \notin U(Z)$, then $P_{Z}(x)$ contains at least two distinct points $z_{1}, z_{2}$. By Lemma 2.5, $\left[z_{1} ; x\right) \subset U(Z)$, implying the density of $U(Z)$ in $X$.

Because $d(x, Z)=d(x, \bar{Z})$, we have $X \backslash U(Z) \subset X \backslash U(\bar{Z})$, so that assertion (2) is a consequence of assertion (3).

Proof of assertion (3). Let $Z$ be a nonempty boundedly compact subset of the strictly convex space $X$ and let

$$
D(x)=\operatorname{diam} P_{Z}(x)=\sup \left\{\left\|z^{\prime}-z^{\prime \prime}\right\|: z^{\prime}, z^{\prime \prime} \in P_{Z}(x)\right\} .
$$


Put $Y=X \backslash U(Z)$ and let

$$
Y_{n}=\left\{x \in X: D(x) \geq \frac{1}{n}\right\}, \quad n \in \mathbb{N}
$$

Since $Y=\bigcup_{n=1}^{\infty} Y_{n}$, it suffices to prove that each set $Y_{n}$ is closed and nowhere dense.

$Y_{n}$ is closed. Let $\left(x_{k}\right)$ be a sequence in $Y_{n}$ converging to an element $x \in X$. Because $Z$ is boundedly compact, the set $P_{Z}\left(x_{k}\right)=Z \cap B\left(x_{k}, d\left(x_{k}, Z\right)\right)$ is compact for every $k \in \mathbb{N}$, so that there exist $z_{k}^{\prime}, z_{k}^{\prime \prime} \in P_{Z}\left(x_{k}\right)$ such that

$$
\left\|z_{k}^{\prime}-z_{k}^{\prime \prime}\right\|=\operatorname{diam} P_{Z}\left(x_{k}\right) \geq \frac{1}{n}
$$

The inequalities $\left\|z_{k}^{\prime}-x\right\| \leq\left\|z_{k}^{\prime}-x_{k}\right\|+\left\|x_{k}-x\right\|, k \in \mathbb{N}$, and similarly for $z_{k}^{\prime \prime}$, show that the sequences $\left(z_{k}^{\prime}\right)$ and $\left(z_{k}^{\prime \prime}\right)$ are bounded. The set $Z$ being boundedly compact, they will contain convergent subsequences. Therefore, without restricting the generality, we can suppose that there exist $z^{\prime}, z^{\prime \prime} \in Z$ such that $z_{k}^{\prime} \rightarrow z^{\prime}$ and $z_{k}^{\prime \prime} \rightarrow z^{\prime \prime}$ for $k \rightarrow \infty$, and $\| z_{k}^{\prime}-$ $z_{k}^{\prime \prime} \| \geq 1 / n, k \in \mathbb{N}$. But then

$$
\left\|z^{\prime}-x\right\|=\lim _{k \rightarrow \infty}\left\|z_{k}^{\prime}-x_{k}\right\|=\lim _{k \rightarrow \infty} d\left(x_{k}, Z\right)=d(x, Z)
$$

and similarly for $z_{k}^{\prime \prime}$ and $z^{\prime \prime}$. It follows $z^{\prime}, z^{\prime \prime} \in P_{Z}(x)$ and $\operatorname{diam} P_{Z}(x) \geq\left\|z^{\prime}-z^{\prime \prime}\right\| \geq 1 / n$, showing that $x \in Y_{n}$, that is, the set $Y_{n}$ is closed.

The set $Y_{n}$ is nowhere dense. As the set $Y_{n}$ is closed, this is equivalent to int $Y_{n}=\varnothing$, where by int $A$ we denote the interior of a set $A$. To prove this, we will use again Lemma 2.5. If $x \in Y_{n}$ then there are $z^{\prime}, z^{\prime \prime} \in P_{Z}(x)$ with $\left\|z^{\prime}-z^{\prime \prime}\right\| \geq 1 / n$. But then, $\left[z^{\prime} ; x\right) \subset U(Z) \subset$ $X \backslash Y_{n}$, so that $Y_{n}$ does not contain any ball with center $x$.

Theorem 2.4 is completely proved.

The following theorem shows that the density result in the above theorem characterizes in fact the strict convexity of $X$.

Theorem 2.6. If $X$ is Banach and $\overline{U(Z)}=X$ for every $Z \subset X$, then $X$ is strictly convex.

Proof. If the space $X$ is strictly convex, then, by Proposition 2.1, the set $U(Z)$ is dense in $X$ for any nonempty subset $Z$ of $X$.

Suppose now that $X$ is not strictly convex, and let $x_{1}, x_{2} \in S_{X}, x_{1} \neq x_{2}$, such that $\| x_{1}+$ $x_{2} \|=2$. Put $x_{0}=\left(x_{1}+x_{2}\right) / 2$ and let $x^{*} \in X^{*}$ be such that $\left\|x^{*}\right\|=1$ and $x^{*}\left(x_{0}\right)=\left\|x_{0}\right\|=$ 1. It follows $x^{*}\left(x_{1}\right)=x^{*}\left(x_{2}\right)=1$. The hyperplane $H=\left\{x \in X: x^{*}(x)=1\right\}$ is proximinal, because $x^{*}$ attains its norm on $S_{X}\left(\right.$ at $\left.x_{0}\right)$. By the well known formula for the distance to a hyperplane, for every $x \notin H, d(x, H)=\left|x^{*}(x)-1\right|$ and $h_{k}=x-\left(x^{*}(x)-1\right) x_{k} \in P_{H}(x)$, $k=1,2$. It follows that $U(H)=H$ is not dense in $X$.

In the presence of local uniform convexity and of uniform convexity, one obtains better topological properties of the sets $U(Z)$ and $E U(Z)$.

THeORem 2.7. If $X$ is locally uniformly convex Banach space, then $X \backslash U(Z)$ is of the first Baire category for any $Z \subset X$. 
In order to present the result in uniformly convex spaces, we recall the notion of well posedness. For $Z \subset X$ and $x \in X$, denote by $\min (x, Z)$ the problem of best approximation of $x$ by elements in $Z$ : find $z_{0} \in Z$ such that $\left\|x-z_{0}\right\|=d(x, Z)$. A sequence $\left(z_{n}\right)$ in $Z$ is called a minimizing sequence if $\lim _{n}\left\|x-z_{n}\right\|=d(x, Z)$. It is clear that minimizing sequences always exist. We say that the problem $\min (x, Z)$ is well posed if it has a unique solution $z_{0} \in Z$ and every minimizing sequence converges to $z_{0}$. A thorough presentation of well posedness in various problems of optimization and optimal control is given in the monograph by Dontchev and Zolezzi [23].

In uniformly convex Banach spaces, the following remarkable result holds.

THEOREM 2.8. If $X$ is a uniformly convex Banach space, then for every nonempty closed subset $Z$ of $X$, the complement of the set of all $x \in X$ for which the problem $\min (x, Z)$ is well posed is of the first Baire category.

For the proofs of these theorems, we need some results on some special subsets of locally uniformly convex and of uniformly convex normed spaces.

Let $x_{0} \in X, r>0,0<\alpha \leq 1 / 2, \beta>0$, and $z \in S\left(x_{0}, r\right)$. Put $x_{1}=x_{0}+\alpha\left(z-x_{0}\right)$ and let

$$
M_{\beta}\left(x_{0}, z ; \alpha\right)=B\left(x_{1},(1-\alpha) r+\beta\right) \backslash B^{\prime}\left(x_{0}, r\right) .
$$

Following De Blasi et al. [19], we call a set of this kind a lens, a term suggested by its geometric shape in the Euclidean space $\mathbb{R}^{3}$.

The inequality from the following proposition will be the key tool in the proofs of uniqueness and existence results in locally uniformly and uniformly convex space.

Proposition 2.9. Let $x_{1}=x_{0}+\alpha\left(x_{0}-z_{0}\right)$ and, for $y \in M_{\beta}\left(x_{0}, z_{0} ; \alpha\right)$, let $z=x_{1}+(1-$ $\alpha) r\left(y-x_{1}\right) /\left\|y-x_{1}\right\|$. Then the following inequality holds:

$$
\left\|y-x_{0}\right\| \leq r+\beta-2 \alpha r \delta
$$

where

$$
\delta=\delta_{X}\left(\frac{\left\|z-z_{0}\right\|}{(1-\alpha) r}, \frac{z_{0}-x_{1}}{(1-\alpha) r}\right) \quad \text { or } \quad \delta=\delta_{X}\left(\frac{\left\|z-z_{0}\right\|}{(1-\alpha) r}\right)
$$

according to the fact that $X$ is locally uniformly convex or uniformly convex.

Proof. Suppose that $X$ is locally uniformly convex. Since $\left\|z_{0}-x_{1}\right\|=\left\|z-x_{1}\right\|=(1-\alpha) r$, we have

$$
\left\|z+z_{0}-2 x_{1}\right\| \leq 2(1-\alpha) r(1-\delta)
$$

where

$$
\delta=\delta_{X}\left(\frac{\left\|z-z_{0}\right\|}{(1-\alpha) r}, \frac{z_{0}-x_{1}}{(1-\alpha) r}\right)
$$


Writing

$$
\begin{aligned}
y-x_{0} & =y-z+z-x_{1}+x_{1}-x_{0}=(y-z)+\left(z-x_{1}\right)+\frac{\alpha}{1-\alpha}\left(z_{0}-x_{1}\right) \\
& =(y-z)+\left(1-\frac{\alpha}{1-\alpha}\right)\left(z-x_{1}\right)+\frac{\alpha}{1-\alpha}\left(z+z_{0}-2 x_{1}\right),
\end{aligned}
$$

we obtain

$$
\left\|y-x_{0}\right\| \leq \beta+\left(1-\frac{\alpha}{1-\alpha}\right)(1-\alpha) r+\frac{2 \alpha}{1-\alpha}(1-\alpha) r(1-\delta)=r+\beta-2 \alpha r \delta .
$$

The proof in the uniformly convex case proceeds in the same way, working with $\delta=$ $\delta_{X}\left(\left\|z-z_{0}\right\| /(1-\alpha) r\right)$.

Proposition 2.10. (1) If $X$ is locally uniformly convex, then

$$
\lim _{\beta \backslash 0} \operatorname{diam} M_{\beta}\left(x_{0}, z ; \alpha\right)=0 .
$$

(2) If $X$ is uniformly convex, then for every $\epsilon>0$, there exists $\gamma=\gamma(\epsilon, \alpha)$ such that

$$
\operatorname{diam} M_{\beta}\left(x_{0}, z ; \alpha\right)<\epsilon
$$

for all $\beta, 0<\beta \leq \gamma$, and all $z \in S_{X}$, that is, $\operatorname{diam} M_{\beta}\left(x_{0}, z ; \alpha\right)$ tends to zero for $\beta \backslash 0$, uniformly with respect to $z \in S_{X}$.

Proof. (1) Suppose, on the contrary, that there exists $\epsilon>0$ and a strictly decreasing sequence of real numbers $\beta_{n} \succ 0$ such that

$$
\operatorname{diam} M_{\beta_{n}}\left(x_{0}, z ; \alpha\right)>2 \epsilon
$$

for all $n \in \mathbb{N}$. Put $M_{n}=M_{\beta_{n}}\left(x_{0}, z ; \alpha\right)$ and choose $y_{n} \in M_{n}$ such that $\left\|y_{n}-z_{n}\right\|>\epsilon$. If $z_{n}^{\prime}=$ $x_{1}+(1-\alpha) r\left\|y-x_{1}\right\|^{-1}\left(y-x_{1}\right)$, then

$$
\left\|z-z_{n}^{\prime}\right\| \geq\left\|z-y_{n}\right\|-\left\|y_{n}-z_{n}^{\prime}\right\| \geq \epsilon-\beta_{n}>\frac{\epsilon}{2},
$$

whenever $\beta_{n}<\epsilon / 2$, which holds for sufficiently large $n$. By Proposition 2.9, we have

$$
\left\|y_{n}-x_{0}\right\| \leq r+\beta_{n}-2 \alpha r \delta
$$

where

$$
\delta=\delta_{X}\left(\frac{\epsilon}{2(1-\alpha) r}, \frac{z-x_{1}}{(1-\alpha) r}\right)
$$

Since $\beta_{n} \backslash 0$ and $\delta$ do not depend on $\beta_{n}$, it follows that $\left\|y_{n}-x_{0}\right\|<r$ for sufficiently large $n$, in contradiction to $y_{n} \in M_{n} \subset X \backslash B^{\prime}\left(x_{0}, r\right)$ (see the definition (2.11) of $M_{n}=$ $\left.M_{\beta_{n}}\left(x_{0}, z ; \alpha\right)\right)$. 
(2) Proceeding again by contradiction, suppose that there exist $\epsilon>0$ and the sequences $\left(z_{n}\right)$ in $S\left(x_{0}, r\right)$ and $\left(\beta_{n}\right)$ in $\mathbb{R}_{+}$with $\beta_{n} \searrow 0$, such that

$$
\operatorname{diam} M_{\beta_{n}}\left(x_{o}, z_{n} ; \alpha\right)>2 \epsilon
$$

for all $n \in \mathbb{N}$. We can suppose $\beta_{n}<\epsilon / 2$. Taking

$$
\delta=\delta_{X}\left(\frac{\epsilon}{2(1-\alpha) r}\right)
$$

and reasoning as above, we obtain

$$
\left\|y_{n}-x_{0}\right\| \leq r+\beta_{n}-2 \alpha r \delta .
$$

It follows $\left\|y_{n}-x_{0}\right\|<r$ for sufficiently large $n$, in contradiction to $y_{n} \notin B^{\prime}\left(x_{0}, r\right)$.

De Blasi et al. [19, Lemma 2.1] gave a quantitative version of Proposition 2.10 and called it Stečkin's lens lemma.

Let $X$ be a uniformly convex space with modulus of uniform convexity $\delta_{X}$. For $\sigma \in$ $(0 ; 1]$, define $\delta^{*}(\sigma)$ by

$$
\delta^{*}(\sigma)=\sup \left\{\epsilon: 0<\epsilon \leq 2, \delta_{X}(\epsilon) \leq \sigma\right\} .
$$

It is immediate that $0<\delta^{*}(\sigma) \leq \delta^{*}\left(\sigma^{\prime}\right)$, for $0<\sigma \leq \sigma^{\prime} \leq 1$, and $\delta^{*}(\sigma) \rightarrow 0$ for $\sigma \searrow 0$.

Proposition 2.11. If $X$ is a uniformly convex space, $x_{0} \in X, r>0,0<\alpha \leq 1 / 2, \beta>0$, and $z \in S\left(x_{0}, r\right)$, then

$$
\operatorname{diam} M_{\beta}\left(x_{0}, z ; \alpha\right) \leq 2 \beta+2(1-\alpha) r \delta^{*}\left(\frac{\beta}{2 \alpha r}\right),
$$

where $M_{\beta}\left(x_{0}, z ; \alpha\right)$ is the lens defined by (2.11).

Proof. Let $y \in M_{\beta}\left(x_{0}, z ; \alpha\right)$ be such that $\|y-z\|>\beta$. If $z^{\prime}=x_{1}+(1-\alpha) r\left(y-x_{1}\right) / \| y-$ $x_{1} \|$, then

$$
\left\|z-z^{\prime}\right\| \geq\|z-y\|-\left\|y-z^{\prime}\right\|>\beta-\beta=0 .
$$

By Proposition 2.9,

$$
r \leq\left\|y-x_{0}\right\| \leq r+\beta-2 r \alpha \delta_{X}\left(\frac{\left\|z-z^{\prime}\right\|}{2 \alpha r}\right),
$$

implying

$$
\delta_{X}\left(\frac{\left\|z-z^{\prime}\right\|}{2 \alpha r}\right) \leq \frac{\beta}{2 \alpha r} .
$$

By the definition of $\delta^{*}$, this inequality yields

$$
\frac{\left\|z-z^{\prime}\right\|}{2 \alpha r} \leq \delta^{*}\left(\frac{\beta}{2 \alpha r}\right)
$$


so that

$$
\begin{gathered}
\left\|z-z^{\prime}\right\| \leq(1-\alpha) r \delta^{*}\left(\frac{\beta}{2 \alpha r}\right), \\
\|y-z\| \leq\left\|y-z^{\prime}\right\|+\left\|z^{\prime}-z\right\| \leq \beta+(1-\alpha) r \delta^{*}\left(\frac{\beta}{2 \alpha r}\right) .
\end{gathered}
$$

Since this inequality trivially holds for $\|y-z\| \leq \beta$, it follows

$$
\left\|y_{1}-y_{2}\right\| \leq\left\|y_{1}-z\right\|+\left\|z-y_{2}\right\| \leq 2 \beta+2(1-\alpha) r \delta^{*}\left(\frac{\beta}{2 \alpha r}\right)
$$

for all $y_{1}, y_{2} \in M_{\beta}\left(x_{0}, z ; \alpha\right)$.

For $x \in X, Z \subset X$, and $\epsilon>0$, let

$$
\begin{gathered}
d(x)=d(x, Z), \quad D(x)=\operatorname{diam} P_{Z}(x) ; \\
P_{Z}^{\epsilon}(x)=\{z \in Z:\|x-z\| \leq \epsilon+d(x)\}=Z \cap B(x, \epsilon+d(x)) ; \\
D_{\epsilon}(x)=\operatorname{diam} P_{Z}^{\epsilon}(x), \quad D_{0}(x)=\lim _{\epsilon \backslash 0} D_{\epsilon}(x) .
\end{gathered}
$$

The quantities $D_{\epsilon}(x)$ and $D_{0}(x)$ are closely related to the well posedness of the problem of best approximation.

The following proposition is taken from De Blasi and Myjak [17].

Proposition 2.12. If $Z$ is a nonempty complete subset of a normed space $X, x \in X$, and $D_{0}(x)=0$, then the problem $\min (x, Z)$ is well posed.

Conversely, if $Z$ is a nonempty subset of $X$ such that the problem $\min (x, Z)$ is well posed, then $D_{0}(x)=0$.

Proof. Suppose that the set $Z$ is complete and $\lim _{\epsilon \backslash 0} D_{\epsilon}(x)=0$. If $\left(z_{n}\right)$ is a minimizing sequence, then for every $\epsilon>0$, there exists $n_{\epsilon}$ such that $z_{n} \in P_{Z}^{\epsilon}(x)$ for all $n \geq n_{\epsilon}$, implying $\left\|z_{n}-z_{m}\right\| \leq \operatorname{diam} P_{Z}^{\epsilon}(x)=D_{\epsilon}(x)$ for all $n, m \geq n_{\epsilon}$. It follows that the sequence $\left(z_{n}\right)$ is fundamental, so that, by the completeness of $Z$, it converges to an element $z_{0} \in Z$ which satisfies $\left\|x-z_{0}\right\|=d(x, Z)$. If $x$ has two distinct elements of best approximation $z_{0}, z_{0}^{\prime}$ in $Z$, then $z_{0}, z_{0}^{\prime}, z_{0} \cdot z_{0}^{\prime}, \ldots$ is a minimizing sequence which is not fundamental, in contradiction with the fact we have just proved.

Suppose now that the problem $\min (x, Z)$ is well posed. If $z_{n}, z_{n}^{\prime} \in P_{Z}^{1 / n}(x)$, then the sequences $\left(z_{n}\right)$ and $\left(z_{n}^{\prime}\right)$ are both minimizing and converge to $z_{0}$ - the solution of the problem, so that $\left\|z_{n}-z_{n}^{\prime}\right\| \rightarrow 0$. It follows $\operatorname{diam} P_{Z}^{1 / n}(x) \rightarrow 0$ for $n \rightarrow \infty$, and $\lim _{\epsilon \backslash 0} \operatorname{diam}$ $P_{Z}^{\epsilon}(x)=0$.

Consider the sets

$$
Y_{n}=\left\{x \in X: D(x) \geq \frac{1}{n}\right\}, \quad F_{n}=\left\{x \in X: D_{0}(x) \geq \frac{1}{n}\right\} .
$$

The sets $Y_{n}$ were used in the proof of Theorem 2.4. Because $P_{Z}(x) \subset P_{Z}^{\epsilon}(x)$, we have $D(x) \leq D_{\epsilon}(x)$, so that $D(x) \leq D_{0}(x)$. It follows $Y_{n} \subset F_{n}$, and $Y_{n}=F_{n}$ if $Z$ is boundedly compact. 
The sets $F_{n}$ have a better topological behavior than the sets $Y_{n}$.

Proposition 2.13. If $X$ is a Banach space, then the set $F_{n}$ is closed for every $n \in \mathbb{N}$.

Proof. Let $\left(x_{k}\right)$ be a sequence in $F_{n}$ converging to an element $x_{0} \in X$. For a given $\epsilon>0$, let $k_{0} \in \mathbb{N}$ be such that $\left\|x_{k}-x_{0}\right\| \leq \epsilon / 3$ for all $k \geq k_{0}$.

Then for all $k \geq k_{0}$,

$$
d\left(x_{k}\right) \leq\left\|x_{k}-x_{0}\right\|+d\left(x_{0}\right) \leq \frac{\epsilon}{3}+d\left(x_{0}\right),
$$

implying

$$
\begin{aligned}
B\left(x_{k}, \frac{\epsilon}{3}+d\left(x_{k}\right)\right) \subset & B\left(x_{k}, \frac{2 \epsilon}{3}+d\left(x_{0}\right)\right) \subset B\left(x_{0}, \epsilon+d\left(x_{0}\right)\right), \\
& P_{Z}^{\epsilon / 3}\left(x_{k}\right) \subset P_{Z}^{\epsilon}\left(x_{0}\right) .
\end{aligned}
$$

It follows

$$
\frac{1}{n} \leq D_{\epsilon / 3}\left(x_{k}\right) \leq D_{\epsilon}\left(x_{0}\right)
$$

Letting $\epsilon>0$, we get $D_{0}\left(x_{0}\right) \geq 1 / n$, that is, $x_{0} \in F_{n}$.

Now we are able to prove Theorem 2.7.

Proof of Theorem 2.7. Let $Z$ be a nonempty subset of the locally uniformly convex space $X$. We have $X \backslash U(Z)=\bigcup_{n=1}^{\infty} Y_{n}$, so it suffices to show that each $Y_{n}$ is nowhere dense in $X$, that is, that int $\overline{Y_{n}}=\varnothing$ for every $n \in \mathbb{N}$. This is equivalent to the fact that $\overline{Y_{n}}$ is not a neighborhood of any point $x \in X$. In its turn, this is equivalent to

$$
\forall x \in X, \forall r>0, \quad \exists y \in B(x, r), \exists r^{\prime}>0 \text { such that } B\left(y, r^{\prime}\right) \cap Y_{n}=\varnothing .
$$

Let $x \in X$ and $r>0$. If $B(x, r) \cap Y_{n}=\varnothing$, then there is nothing to prove. Suppose that there is an element $x_{0} \in B(x, r) \cap Y_{n}$. It follows $x_{0} \notin Z$.

If $z_{0} \in P_{Z}\left(x_{0}\right)$ and $y \in\left(x_{0} ; z_{0}\right)$, then, by Lemma $2.5, P_{Z}(y)=\left\{z_{0}\right\}$ and $d(x, Z)=\| x_{0}-$ $y \|+d(y, Z)$. For $\epsilon>0$,

$$
P_{Z}^{\epsilon}(y) \subset B(y, \epsilon+d(y, Z)) \backslash B^{\prime}\left(x_{0}, d\left(x_{0}, Z\right)\right)=M_{\epsilon}\left(x_{0}, z_{0} ; \alpha\right),
$$

where $\alpha=\left\|y-x_{0}\right\| /\left\|z_{0}-x_{0}\right\|$.

We have $D_{\epsilon}(y)=\operatorname{diam} P_{Z}^{\epsilon}(y) \leq \operatorname{diam} M_{\epsilon}\left(x_{0}, z_{0} ; \alpha\right)$, so that, by Proposition 2.10, $D_{\epsilon}(y)$ $\rightarrow 0$ for $\epsilon \backslash 0$. It follows $D_{0}(y)=0<1 / n$, showing that $y \notin F_{n}$. But, by Proposition 2.13, the set $X \backslash F_{n}$ is open, so that there exists $r^{\prime}>0$ such that $B\left(y, r^{\prime}\right) \subset X \backslash F_{n} \subset X \backslash Y_{n}$. Theorem 2.7 is proved.

Theorem 2.8 is an immediate consequence of the following result from [76] and of Proposition 2.12.

THeOREM 2.14. If $X$ is a uniformly convex Banach space, then for every nonempty subset $Z$ of $X$, the set $\left\{x \in X: D_{0}(x)=0\right\}$ is $G_{\delta}$ and dense in $X$. 
Proof. If $F_{0}=\left\{x \in X: D_{0}(x)=0\right\}$, then $F_{0}=\bigcap_{n=1}^{\infty}\left(X \backslash F_{n}\right)$, so that

$$
X \backslash F_{0}=\bigcup_{n=1}^{\infty} F_{n} .
$$

Since, by Proposition 2.13, every set $F_{n}$ is closed, we have only to show that the set $F_{n}$ is nowhere dense for every $n \in \mathbb{N}$, meaning that $B(x, r) \cap\left(X \backslash F_{n}\right) \neq \varnothing$, for every $x \in F_{n}$ and every $r>0$. For $x \in F_{n}$, let $d=d(x, Z)>0$ and consider a ball $B(x, \alpha d)$, with $0<\alpha \leq 1 / 2$. By Proposition 2.10, there exists $\beta_{0}>0$ such that

$$
\operatorname{diam} M_{\beta}(x, y ; \alpha)<\frac{1}{n}
$$

for all $\beta, 0<\beta \leq 2 \beta_{0}$, and all $y \in S(x, \alpha d)$. Let $z \in P_{Z}^{\beta_{0}}(x)$, that is, $z \in Z$ and $d \leq\|x-z\| \leq$ $d+\beta_{0}$, and put $y=x+\alpha d z /\|z\|$. We have

$$
d(y, Z) \leq\|y-z\|=\|x-z\|-\|x-y\| \leq(1-\alpha) d+\beta_{0},
$$

implying

$$
P_{Z}^{\beta_{0}}(y) \subset M_{2 \beta_{0}}(x, y ; \alpha)
$$

It follows $\operatorname{diam} P_{Z}^{\beta_{0}}(y) \leq \operatorname{diam} M_{2 \beta_{0}}(x, y ; \alpha)<1 / n$, that is, $y \in B(x, \alpha d) \cap\left(X \backslash F_{n}\right)$.

The following problems were raised in Stečkin's paper [76]. A normed space $X$ is called strongly convex if

$$
\lim _{t \rightarrow d} \operatorname{diam}\left(Z \cap t B_{X}\right)=0
$$

for any convex subset $Z$ of $X$, where $d=d(0, Z)$ and $B_{X}$ denotes the closed unit ball of $X$.

Stečkin's problems. (1) Does assertion (2) of Theorem 2.4 remain true for an arbitrary subset $Z$ of $X$ ? Also, if $X$ is Banach and $\overline{U(Z)}=X$ (or $X \backslash U(Z)$ is of the first Baire category) for every compact subset $Z$, then must $X$ be strictly convex?

(2) Is Theorem 2.7 true for strongly convex Banach spaces?

(3) Is the conclusion of Theorem 2.8 true for locally uniformly convex Banach spaces?

We mention also some related existence results obtained by Borwein and Fitzpatrick [9].

Theorem 2.15 (see [9]). Let $X$ be a reflexive Banach space. Then the following assertions hold:

(1) the complement of every open convex proper subset of $X$ is almost proximinal,

(2) if $\left\{U_{i}: i \in I\right\}$ is a collection of mutually disjoint open convex subsets of $X$, then the set $X \backslash \bigcup\left\{U_{i}: i \in I\right\}$ is almost proximinal in $X$, provided it is nonempty.

Approximative compactness and Efimov-Stečkin spaces. A reflexive Banach space having the Kadec-Klee property is called an Efimov-Stečkin (ES) space. 
A point $x \in X$ is called a point of approximative compactness for $Z \subset X$ if every minimizing sequence for $d(x, Z)$ contains a convergent subsequence. The set of points of approximative compactness for the set $Z$ is denoted by $A C(Z)$. The set $Z$ is called approximatively compact if $A C(Z)=X$.

The notion of approximative compactness was introduced by Efimov and Stečkin [31], in connection with the problem of convexity of Chebyshev sets. It was also used by Reich [68] to study continuity properties of the metric projection with applications to fixed point theorems of Ky Fan type.

Although, at the first glance, this notion looks a little tautological, it turned to be a very useful one in the study best approximation problems. It differs from the notion of compactness: Borodin [6] has shown that every separable infinite-dimensional Banach space contains a bounded approximatively compact set which is not compact. In [7], he showed that every reflexive Banach space contains a noncompact bounded convex approximatively compact set.

Efimov and Stečkin proved the following.

Theorem 2.16. Let $X$ be a uniformly convex smooth Banach space and $Z$ a Chebyshev subset of $X$.

The set $Z$ is convex if and only if it is approximatively compact.

The term Efimov-Stečkin space was proposed by Singer [74].

There are several conditions equivalent to the ES property.

Theorem 2.17 (see Singer [74] and Konyagin and Tsar'kov [49]). For a Banach space X, the following conditions are equivalent:

(1) $X$ is an ES space;

(2) $X$ is reflexive and Kadec-Klee;

(3) any sequentially closed subset of $X$ is compact;

(4) every weakly closed subset of $X$ is compact.

There are also some characterizations of ES spaces in terms of approximative compactness.

Theorem 2.18 (see Singer [74] and Konyagin and Tsar'kov [49]). For a Banach space X, the following conditions are equivalent:

(1) $X$ is an ES space;

(2) every weakly sequentially closed subset of $X$ is approximatively compact;

(3) every weakly closed subset of $X$ is approximatively compact;

(4) every closed convex set is approximatively compact;

(5) every weakly sequentially closed set is an existence set with lower semicontinuous metric projection;

(6) every weakly closed set is an existence set with lower semicontinuous metric projection;

(7) every weakly sequentially closed set is an existence set with lower semicontinuous metric projection;

(8) every closed convex set is an existence set with lower semicontinuous metric projection.

Condition (2) from the above theorem was taken by Singer [74] as the definition of ES spaces. 
$E S$ spaces are also important in the study of generic existence for best approximation. Denote by $\mathscr{P}_{\mathrm{cl}}(X)$ the family of all nonempty closed subsets of a normed space $X$.

Theorem 2.19 (see Konyagin and Tsar'kov [49]). For a Banach space X, the following conditions are equivalent:

(1) $X$ is an ES space;

(2) for all $Z \in \mathscr{P}_{\mathrm{cl}}(X)$, the set $A C(Z)$ is residual in $X$;

(3) for all $Z \in \mathscr{P}_{\mathrm{cl}} B(X)$, the set $A C(Z)$ is dense in $X$;

(4) for all $Z \in \mathscr{P}_{\mathrm{cl}}(X)$, the set $E(Z)$ is residual in $X$;

(5) for all $Z \in \mathscr{P}_{\mathrm{cl}}(X)$, the set $E(Z)$ is dense in $X$;

(6) for all $Z \in \mathscr{P}_{\mathrm{cl}}(X)$, the set $E(Z)$ is weakly dense in $X$;

(7) for all $Z \in \mathscr{P}_{\mathrm{cl}} B(X)$, the set $A C(Z)$ is connex;

(8) for all $Z \in \mathscr{P}_{\mathrm{cl}}(X)$, the set $E(Z)$ connex.

(9) for every $Z \in \mathscr{P}_{\mathrm{cl}}(X)$, there exists a residual set $A$ such that $A \subset E(Z)$ and the setvalued map $P_{Z} \mid A$ is continuous;

(10) for every $Z \in \mathscr{P}_{\mathrm{cl}} B(X)$, there exists a residual set $B$ such that the set-valued map $P_{Z}$ has a continuous selection on $B$.

Strongly convex spaces. This notion was introduced by Šmulian [75] and, for further properties, one can consult also the paper by Fan and Glicksberg [32]. As we have already mentioned, Stečkin used it in his paper [76] on approximation properties of Banach spaces. Recall that a Banach space $X$ is called strongly convex if $\lim _{t \rightarrow d} \operatorname{diam}\left(Z \cap t B_{X}\right)=0$, for any convex subset $Z$ of $X$.

Sometimes the strong convexity (or strong rotundity) is denoted by $(K)$, the fact that $X$ is Banach and satisfies $(K)$ is denoted by $(\mathrm{D})$, and the Kadec-Klee property by $(\mathrm{H})$. In fact, in [32], there is a list of geometric properties that a normed space could satisfy, labelled from $(\mathrm{A})$ to $(\mathrm{H})$, and the Kadec-Klee property is labelled as $(\mathrm{H})$.

The following proposition puts in evidence some connections of this notion with other geometric properties of Banach spaces.

THEOREM 2.20. The following assertions hold:

(1) if $X$ is uniformly convex, then $X$ is strongly convex;

(2) if $X$ is reflexive and locally uniformly convex, then $X$ is strongly convex;

(3) if $X$ is strongly convex, then $X$ is strictly convex and Kadec-Klee,

(4) if $X$ is Banach, then $X$ is strongly convex if and only if it is reflexive strictly convex and Kadec-Klee.

For a good presentation of these results and of other geometric properties of Banach spaces, one can consult, for instance, Megginson [58, Section 5.3].

The following theorem contains some equivalent characterizations of strongly convex spaces.

Theorem 2.21. For a normed space $X$, the following conditions are equivalent:

(1) $X$ is strongly convex;

(2) for every $x^{*} \in S_{X^{*}}, \lim _{\epsilon \rightarrow 0+} \operatorname{diam}\left\{x \in B_{X}: x^{*}(x) \geq 1-\epsilon\right\}=0$;

(3) if $\left(x_{n}\right) \subset S_{X}$ and $x^{*}\left(x_{n}\right) \rightarrow 1$, for some $x^{*} \in S_{X^{*}}$, then $\left(x_{n}\right)$ is a Cauchy sequence; 
(4) for every convex subset $Z$ of $X$ and $x \in X$, every minimizing sequence $\left(z_{n}\right) \subset Z$ is a Cauchy sequence.

If $X$ is Banach, then the above conditions are also equivalent to the following one:

(5) every nonempty closed convex subset of $X$ is an approximatively compact Chebyshev set.

Some definitive results concerning the problems raised by Stečkin were obtained by Konyagin [42, 43, 44, 45, 46, 47] and Konyagin and Tsar'kov [49]. A good account is given in the survey paper [48]. In the following theorem, we collect some of these results.

Theorem 2.22. For a Banach $X$, the following conditions are equivalent:

(1) for all $Z \subset X$, the set $E(Z)$ is dense in $X$;

(2) for all $Z \subset X$, the set $E(Z)$ is residual in $X$;

(3) for all $Z \subset X$, the set $A C(Z)$ is dense in $X$;

(4) for all $Z \subset X$, the set $A C(Z)$ is residual in $X$;

(5) $X$ is an ES space.

The implication $(5) \Rightarrow(1)$ was proved by Lau [51] and the implication (1) $\Rightarrow(5)$ by Konyagin [42]. The others are trivial.

Konyagin [42] (see also [9]) proved the following result.

Theorem 2.23. Let $X$ be a Banach space that is neither reflexive nor Kadec-Klee. Then there exists a closed bounded set $Z$ of $X$ and an open nonempty subset $U$ of $X \backslash Z$ such that

(i) no $x \in U$ has a nearest point in $Z$;

(ii) the distance function $d(\cdot, Z)$ is affine on $U$, so that, in particular, $d(\cdot, Z)$ is Fréchet differentiable on $U$.

Konyagin [42] has also proved.

Theorem 2.24. For a Banach space $X$, the following conditions are equivalent:

(1) for all $Z \subset X, E U(Z)$ is dense in $X$;

(2) for all $Z \subset X, E U(Z)$ is residual in $X$;

(3) $X$ is strictly convex and $E S$.

A point $x$ of a closed bounded convex subset $Z$ of a Banach space $X$ is called an exposed point of $Z$ if $x$ is the only point of $Z$ for which $x^{*}(x)=\sup x^{*}(Z)$, for some $x^{*} \in X^{*}$, $x^{*} \neq 0$. One says that the functional $x^{*}$ exposes the point $x$. The point $x$ is called a strongly exposed point of $Z$ if it is an exposed point of $Z$ with exposing functional $x^{*}$ and, for every sequence $\left(z_{n}\right)$ in $Z$ such that $x^{*}\left(z_{n}\right) \rightarrow \sup x^{*}(Z)$, we have $z_{n} \rightarrow x$ in the norm topology of $X$. This is equivalent to $\operatorname{diam} S\left(Z, x^{*}, \epsilon\right) \rightarrow 0$ for $\epsilon \rightarrow 0+$, where, for $\beta=\sup x^{*}(Z)$, $S\left(Z, x^{*}, \epsilon\right)=\left\{z \in Z: x^{*}(z)>\beta-\epsilon\right\}$. The notion of exposed point is due to Straszewicz [77].

The notion of exposed point is closely related to two other geometric properties of Banach spaces - the Radon-Nikodým property and Asplund property. Let $(\Omega, \mathcal{A}, \mu)$ be a finite measure space, that is, $\Omega$ is a set, $\mathscr{A}$ a $\sigma$-algebra of subsets of $\Omega$, and $\mu: \mathscr{A} \rightarrow[0 ; \infty)$ a $\sigma$-additive finite positive measure on $\mathscr{A}$. One says that a Banach space $X$ has the RadonNikodym property with respect to the measure space $(\Omega, \mathcal{A}, \mu)$ if for every $\sigma$-additive vector measure $G: \mathscr{A} \rightarrow X$, there exists $g \in L^{1}(\mu, X)$ such that $G(A)=\int_{A} g d \mu, A \in \mathscr{A}$. The 
Banach space $X$ has the Radon-Nikodým property if it has the Radon-Nikodým property with respect to every finite measure space $(\Omega, \mathcal{A}, \mu)$. The classic on Radon-Nikodým property is the book by Diestel and Uhl [22] (see also [11]). A Banach space $X$ is said to have the Asplund property if every continuous convex function defined on a nonempty open subset $Z$ of $X$ is Fréchet differentiable on a dense $G_{\delta}$ subset of $Z$. The term was chosen to honor Asplund [2], who made important contributions to the subject. A good presentation of the results concerning differentiability properties of convex functions on Banach spaces can be found in the books $[33,67]$.

Denote by near $(Z)$ the set of all $z \in Z$ such that $\|x-z\|=d(x, Z)$ for some $x \in X$. Let also $\operatorname{spt}(Z)$ denote the set of all support points of the set $Z$, that is, those points $z \in Z$ for which there exists a nonzero $x^{*} \in X^{*}$ such that $x^{*}(z)=\sup x^{*}(Z)$. It follows $\operatorname{near}(Z) \subset \operatorname{spt}(Z) \subset \operatorname{bd}(Z)$.

The problem of the existence of convex sets $Z$ such that near $(Z) \neq \operatorname{spt}(Z)$ was investigated by Godini [34], Borwein and O'Brien [10], and Borwein [8].

Theorem 2.25. If the Banach space $X$ has the Radon-Nikodým property, then

(1) $\overline{\mathrm{co}}(\operatorname{near}(Z))=Z$ for every closed convex subset $Z$ of $X$,

(2) the same relation holds for any weakly compact convex subset $Z$ of an arbitrary $B a-$ nach space $X$.

Borwein and O'Brien [10] constructed an example of a proximinal set $Z$ in a nonreflexive Banach space $X$ such that $\operatorname{bd}(Z)=\operatorname{spt}(Z) \neq$ near $(Z)$, where bd stands for the topological boundary of a set in a topological space.

A Banach space $X$ is said to have the property $(\sigma)$ if every closed bounded convex subset of $X$ is the closed convex hull of its strictly exposed points. A remarkable result in the Banach space theory says that a Banach space $X$ has the property $(\sigma)$ if and only if it has the Radon-Nikodým property (see [67, Theorem 5.21]). A point $x^{*}$ of a subset $Z$ of a conjugate Banach space $Y=X^{*}$ is called strongly $w^{*}$-exposed if there exists $x \in X$ such that $x^{*}(x)=\sup Z(x)$ and if $\left(z_{n}^{*}\right)$ in $Z$ satisfies $z_{n}^{*}(x) \rightarrow x^{*}(x)$, then $z_{n}^{*} \rightarrow x^{*}$ in the norm topology. We mention also the following important result: a Banach space $X$ is an Asplund space if and only if every nonempty $w^{*}$-compact convex subset of $X^{*}$ is the $w^{*}$-closed convex hull of its strongly $w^{*}$-exposed points (see [67, Theorem 5.12]). Since a Banach space $X$ is an Asplund space if and only if its dual space $X^{*}$ has the RadonNikodým property (see [67, Theorem 5.7]), it follows that the dual of an Asplund space has the property $(\sigma)$, a result contained in [25, Proposition 2.2].

Some interesting connections between the existence of nearest points and of strongly exposed points was established by Edelstein [25].

THEOREM 2.26. If $Z$ is a closed bounded convex subset of a Banach space having the property $(\sigma)$, then the set $E(Z)$ of all $x \in X$ having a nearest point in $Z$ is weakly dense in $X$.

Moreover, $E(Z)$ contains infinitely many rays emanating from points of $Z$.

The actual status of Stečkin's problems. Concerning problem (3), it was shown by Cobzaş $[12,13]$ (see also [26]) that the space $c_{0}$ equipped with Day's locally uniformly convex norm contains a closed bounded symmetric antiproximinal convex body $Z$, that is, such that no point outside $Z$ has a nearest point in $Z$, showing that Theorem 2.8 does not hold, in general, for locally uniformly convex Banach spaces. A complete solution to this 
problem and to the related one, problem (2), was given by Konyagin: strongly convex Banach spaces are exactly those for which Theorem 2.8 is true.

Problem (2) is still open. We will expose, following [48], some progress made towards the solution of "the most intriguing of the Stečkin's problems," as Konyagin calls it in [48], and on the closely related problem (1). There are some particular cases when the answer to problem (2) is yes as, for instance, if the strongly convex space $X$ is separable (see $[41,79]$ ), or if there exists a Fréchet differentiable bump function on $X$ (see [42]). These results were obtained independently by Zhivkov $[83,84]$, who proved that the answer is yes for a class of strictly convex Banach spaces, containing the weakly compactly generated Banach spaces. Stečkin [76] proved that if $Z$ is a boundedly compact subset of a strictly convex Banach space $X$, then $X \backslash U(Z)$ is of the first Baire category. Kenderov [38, 39, 40] extended this result to more general classes of subsets by proving it for approximatively compact subsets of a strictly convex Banach space. Konyagin [42] proved that in a strictly convex Banach space, the set $A C(Z) \backslash U(Z)$ is of the first Baire category for any subset $Z$, but this property does not characterize strictly convex Banach spaces, because, as it was shown by Kamuntavichyus [36], this property holds in the space $L^{1}(\Omega, \mathcal{A}, \mu)$ with a nonatomic measure $\mu$, and these spaces are not strictly convex.

\section{The problem of farthest points}

Generic existence of farthest points for closed bounded sets was proved by Edelstein [24] for uniformly convex spaces, by Asplund [1] for reflexive locally uniformly convex spaces, and by Lau [50] for weakly compact subsets of arbitrary Banach spaces.

Theorem 3.1 (see Lau [50]). If $Z$ is a weakly compact subset of a Banach space $X$, then the set $X \backslash e(Z)$ is of the first Baire category.

Deville and Zizler [21] proved a kind of converse of this result.

Theorem 3.2. Let $X$ be a Banach space and $Z$ a closed convex subset of $X$. If the set $Z$ is almost $\|\cdot\|_{1}$-remotal for any equivalent norm $\|\cdot\|_{1}$ on $X$, then the set $Z$ is weakly compact.

Edelstein and Lewis [27] have shown that there exists a closed bounded convex subset $Z$ of $\ell_{2}$ such that no point in the dense subspace $\ell_{0}$, of all finitely supported sequences, has a farthest point in $Z$. Also no point in $\ell_{0}$ has a farthest point in $Z \cap \ell_{0}$.

Interesting connections between the existence of farthest points and Mazur intersection property were established. One says that a Banach space $X$ has the Mazur intersection property (MIP in short) if any bounded closed convex subset of $X$ can be written as an intersection of closed balls. Mazur [57] proved that MIP holds in finite-dimensional Euclidean spaces. Phelps [66] gave a dual characterization of finite-dimensional Banach normed spaces which have the MIP and proved that Banach spaces with Fréchet differentiable norm have the MIP, resuscitating the interest to this important geometric property. The property turned out to have very interesting connections with other geometric properties of Banach spaces, from which we will emphasize only those related to the existence of farthest points.

As in the case of nearest points, denote by $\operatorname{far}(Z)$ the set of all points $z \in Z$ such that $z \in Q_{Z}(x)$ for some $x \in X$. Recall that $Q_{Z}(x)$ denotes the set of farthest points from $x$ in $Z$. 
Theorem 3.3. (1) If $X$ has MIP, then $\overline{\mathrm{co}}(\operatorname{far}(Z))=Z$ for any $w$-compact convex subset $Z$ of $X$.

(2) If $X$ is reflexive, then $X$ has MIP if and only if $\overline{\operatorname{co}} \operatorname{far}(Z)=Z$ for any closed convex set $Z \subset X$.

Remark 3.4. The first assertion of the above theorem was proved by Edelstein [24] and the second one by Lau [50]. Some extensions of these results as well as a discussion on the relations between $w^{*}$-MIP and the existence of farthest points of $w^{*}$-compact subsets of the dual space $X^{*}$ of $X$ were done by Bandyopadhyay [4].

Concerning the existence of farthest points of $w^{*}$-compact subsets of dual Banach spaces, we mention the following result of Deville and Zizler [21].

Theorem 3.5. If the Banach space $X$ has the Radon-Nikodym property, then every $w^{*}$ compact subset of $X^{*}$ is almost remotal.

As in the case of nearest points, the strict convexity of the space $X$ guarantees the density of the elements having a unique farthest point in a subset. More exactly, the following theorem holds.

Theorem 3.6 [21]. Suppose that $X$ is a strictly convex Banach space and $Z$ is a norm closed bounded subset of $X$, such that the set $e(Z)$ is dense in $X$. Then the set eu $(Z)$ is also dense in $X$.

Another interesting question is that of the relations between farthest points and exposed points. In a locally uniformly convex Banach space, every farthest point of $Z$ is a strongly exposed point of $Z$, and this was the method used by Straszewicz [77] to obtain exposed points of sets. Also, in a smooth Banach space $X$, we have $\exp (Z) \subset \operatorname{far}(Z)$, for any compact convex subset $Z$ of $X$. The relations between farthest points and exposed point is complicated enough, as was shown by Bernau [5] and Edelstein and Lewis [27]. Bernau [5] has shown that every normed space of dimension greater than one contains a compact convex set $Z$ for which $\exp (Z)$ is not a subset of $\operatorname{far}(Z)$. Edelstein and Lewis [27] constructed two-dimensional compact convex sets $Z$ with $\operatorname{far}(Z) \cap \exp (Z)=\varnothing$.

Deville and Zizler [21] proved that the set

$$
Z=\left\{x \in \ell^{1}: \sum_{n=1}^{\infty}\left(\left|x_{n}\right|+\left|x_{n}\right|^{2}\right) \leq 1\right\}
$$

is a $w^{*}$-compact convex subset of the Banach space $\ell^{1}$ which has no farthest points in $\ell^{1}$, that is, $Q_{Z}(x)=\varnothing$ for all $x \in \ell^{1}$. Since the space $\ell^{1}$ has the Radon-Nikodým property, the set $Z$ is the closed convex hull of its strongly exposed points, so it furnishes an example of a set having strongly exposed points but without farthest points. For results concerning strongly exposed points and Radon-Nikodým property, one can consult the book by Bourgin [11].

A bounded subset $Z$ of a normed space $X$ is called uniquely remotal if, for all $x \in X$, card $Q_{Z}(x)=1$, that is, any point in $X$ has a unique farthest point in $Z$. An important problem in the study of farthest points is the following one: under what conditions on the space $X$ is every uniquely remotal subset of $X$ a singleton? This problem is closely 
connected with the problem of convexity of Chebyshev sets. Both these problems are unsolved till now, and there are some opinions (see, e.g., [61]) that the solution of one of them will lead to the solution of the other one too. For instance, and this is the most challenging question, it is unknown whether every Chebyshev subset of a Hilbert space must be convex. A new approach was proposed by Ricceri in [73] (see also [37]). Good survey papers on the convexity of Chebyshev sets are those by Vlasov [78], Balaganskil and Vlasov [3], and Narang [60], and, for the problem of farthest points, Narang [59, 61].

\section{Other results}

De Blasi, Myjak, and Papini [20] extended the problems of nearest and farthest points to subsets $A, Z \subset X$. Let

$$
\begin{aligned}
& \lambda_{A Z}=\inf \{\|x-z\|: x \in A, z \in Z\}, \\
& \mu_{A Z}=\sup \{\|x-z\|: x \in A, z \in Z\} .
\end{aligned}
$$

The problem $\min (A, Z)$ (resp., $\max (A, Z))$ consists in finding $x_{0} \in A$ and $z_{0} \in Z$ such that $\left\|x_{0}-z_{0}\right\|=\lambda_{A Z}$ (resp., $\left\|x_{0}-z_{0}\right\|=\mu_{A Z}$ ).

Denote by $\mathscr{B}(X)$ the family of all nonempty closed bounded subsets of the normed space $X$, by $\mathscr{C}(X)$ the family of all nonempty closed bounded convex subsets of $X$, and by $\mathscr{K}(X)$ the family of all nonempty compact convex subsets of $X$. If $X$ is a Banach space, then $\mathscr{B}(X), \mathscr{C}(X)$, and $\mathscr{K}(X)$ are all complete with respect to the Pompeiu-Hausdorff metric $H$ (see [35]). For $A \in \mathscr{B}(X)$, let

$$
\mathscr{B}_{A}(X)=\operatorname{cl}\left\{Z \in \mathscr{B}(X): \lambda_{A Z}>0\right\},
$$

where the closure $\mathrm{cl}$ is taken with respect to the Pompeiu-Hausdorff metric $H$, and let $\mathscr{C}_{A}(X)=\mathscr{C}(X) \cap \mathscr{B}_{A}(X)$. In the following, all the topological notions concerning the spaces $\mathscr{B}(X), \mathscr{C}(X), \mathscr{K}(X)$ will be considered with respect to the Pompeiu-Hausdorff metric $H$.

One says that the problem $\min (A, Z)$ is well posed if it has a unique solution $\left(y_{0}, z_{0}\right)$ and every minimizing sequence (i.e., a sequence $\left(y_{n}, z_{n}\right) \in A \times Z$ such that $\left.\left\|y_{n}-z_{n}\right\| \rightarrow \lambda_{A, Z}\right)$ converges to $\left(y_{0}, z_{0}\right)$. The well posedness of the problem $\max (A, Z)$ is defined similarly.

The following results were proved in [20].

Theorem 4.1. Let $X$ be a uniformly convex Banach space.

(1) For $A \in \mathscr{B}_{B}(X)$, the set of all $Z \in \mathscr{C}_{A}(X)$ for which the problem $\min (A, Z)$ is well posed is $G_{\delta}$ and dense in $\mathscr{C}_{A}(X)$.

(2) For $A \in \mathscr{B}(X)$, the set of all $Z \in \mathscr{C}(X)$, for which the problem $\max (A, Z)$ is well posed is $G_{\delta}$ and dense in $\mathscr{C}(X)$.

Li [52] proved similar results for the family $\mathscr{K}(X)$ of all nonempty compact convex subsets of a strongly convex Banach spaces $X$. Recall that, by Theorem 2.20(4), a Banach space is strongly convex if and only if it is reflexive, strictly convex, and Kadec-Klee.

For $A \in \mathscr{B}(X)$, put $\mathscr{K}_{A}(X)=\mathscr{K}(X) \cap \mathscr{S}_{A}(X)$. 
Theorem 4.2 [52]. Let $X$ be a strongly convex Banach space.

(1) If $A$ is a nonempty closed subset of $X$, then the set of all $Z \in \mathscr{K}_{A}(X)$ for which the problem $\min (A, Z)$ is well posed contains a dense $G_{\delta}$ subset of $\mathscr{K}_{A}(X)$.

(2) If $A$ is a nonempty closed bounded subset of $X$, then the set of all $Z \in \mathscr{K}(X)$ for which the problem $\max (A, Z)$ is well posed contains a dense $G_{\delta}$ subset of $\mathscr{Y}(X)$.

Li [52] has also shown that the property (1) in the above theorem characterizes strongly convex spaces.

Theorem 4.3. Let $X$ be a Banach space. If for every nonempty closed set $A$ the set of all $Z \in \mathscr{K}_{A}(X)$ for which the problem $\min (A, Z)$ is well posed contains a dense $G_{\delta}$ subset of $\mathscr{K}_{A}(X)$, then the space $X$ is strongly convex.

The same is true with " $G_{\delta}$ dense" replaced by "dense" in the above assertion.

Supposing the set $A$ relatively boundedly weakly compact (i.e., its intersection with any ball is relatively weakly compact), $\mathrm{Li}$ and $\mathrm{Xu}$ [54] succeeded in dispensing with the reflexivity of the space $X$.

Theorem 4.4. Let $X$ be a strictly convex Banach space satisfying the Kadec-Klee condition.

(1) If $A$ is a relatively boundedly compact subset of $X$, then the family of all $Z \in \mathscr{B}_{A}(X)$ for which the problem $\min (A, Z)$ is well posed is a $G_{\delta}$ dense subset of $\mathscr{B}_{A}(X)$.

The same is true for the family $\mathcal{K}_{A}(X)$.

(2) If $A$ is a nonempty closed relatively weakly compact bounded subset of $X$, then the family of all $Z \in \mathscr{M}_{A}(X)$ for which the problem $\max (A, Z)$ is well posed is a dense $G_{\delta}$ subset of $\mathscr{B}_{A}(X)$.

The same is true for the family $\mathscr{K}(X)$.

Porosity results. Many of the above generic existence results can be strengthened by using the notion of porosity. A subset $Z$ of a metric space $X$ is called porous if there exist $\alpha$, $0<\alpha \leq 1$, and $r_{0}>0$ such that for every $x \in X$ and $0<r \leq r_{0}$, there is $y \in X$ such that $B^{\prime}(y, \alpha r) \subset B^{\prime}(x, r) \backslash Z$. Here $B^{\prime}(x, r)$ denotes the open ball of center $x$ and radius $r$. One obtains the same thing if we replace in the above definition "for every $x \in X$ " by "for every $x \in Z$." A set $Z$ is called $\sigma$-porous if it is a countable union of porous subsets of $X$. A porous set is nowhere dense in $X$, so that a $\sigma$-porous set is of the first Baire category. In general (e.g., if $X$ is a Banach space), the family of sets of the first Baire category strictly contains the family of all $\sigma$-porous sets and, if $X=\mathbb{R}^{n}$, then a $\sigma$-porous set is also of Lebesgue measure zero. Since $\mathbb{R}^{n}$ can be written as the union of a set of the first Baire category and of a set of Lebesgue measure zero, it follows that there are sets of Lebesgue measure zero which are not $\sigma$-porous. A good account of various questions related to porosity is given in the survey papers by Zajíček $[80,81]$. Remark that for what we call here "porous," Zajíček uses the term "uniformly very porous," in his definition of porosity, the number $\alpha$ may depend on $x$.

De Blasi et al. [19] proved porosity existence results for nearest- and farthest-point problems in uniformly convex Banach spaces, extending some known generic existence results.

As in the case of the problem of nearest points, one says that, for $x \in X$ and a bounded subset $Z$ of $X$, the problem of farthest points $\max (x, Z)$ is well posed if it has a unique 
solution $z(x)$ and every maximizing sequence $\left(z_{n}\right) \subset Z$ converges to $z(x)$. Denote by $\operatorname{nwp}(Z)$ and $\operatorname{fwp}(Z)$ the set of all points $x \in X$ for which the problem of nearest points (resp., of farthest points) is well posed.

A result similar to Proposition 2.12 holds for the problem of farthest points too. Suppose that $(e(x, Z)=\sup \{\|x-z\|: z \in Z\}>0$. For $0<\epsilon<e(x, Z)$, let

$$
Q_{Z}^{\epsilon}(x)=Z \cap B(x, e(x, Z)-\epsilon)
$$

Proposition 4.5 [17]. For a Banach space $X$, a bounded subset $Z$ of $X$, and $x \in X$, the problem $\max (x, Z)$ is well posed if and only if $\lim _{\epsilon \backslash 0} \operatorname{diam} Q_{Z}^{\epsilon}(x)=0$.

De Blasi et al. [19] proved the following result.

Theorem 4.6. Let $X$ be a uniformly convex Banach space.

(1) If $Z$ is a nonempty closed subset of $X$, then $X \backslash \operatorname{nwp}(Z)$ is a $\sigma$-porous subset of $X$.

(2) If $Z$ is nonempty bounded closed subset of $X$, then $X \backslash \mathrm{fwp}(Z)$ is a $\sigma$-porous subset of $X$.

Lau [51] proved that for very closed subset $Z$ of a reflexive Banach space $X$ satisfying the Kadec-Klee property, the set $E(Z)$ is $G_{\delta}$ and dense in $X$. Concerning the problem of farthest points, he proved in [50] that for very weakly compact subset $Z$ of an arbitrary Banach space $X$, the set of all points in $X$ having a farthest point in $Z$ is $G_{\delta}$ and dense in $X$.

In connection with these results, the following questions naturally arise.

Problems. (1) Does the first assertion of Theorem 4.6 remain true for a reflexive Banach space satisfying the Kadec-Klee property?

(2) The same question is for the second assertion and a weakly compact subset of an arbitrary Banach space $X$.

$\mathrm{Li}$ and $\mathrm{Xu}$ [54] proved some porosity results for the well posedness with respect to families of subsets of a Banach space equipped with the Pompeiu-Hausdorff metric.

Theorem 4.7 (see De Blasi et al. [19]). Let X be a uniformly convex Banach space.

(1) If $A$ is a nonempty closed subset of $X$ and $\operatorname{minwp}(A)$ denotes the family of all sets $Z \in \mathscr{B}_{A}(X)$ for which the problem $\min (A, Z)$ is well posed, then the set $\mathscr{B}_{A}(X) \backslash$ $\operatorname{minwp}(A)$ is $\sigma$-porous in $\mathscr{B}_{A}(X)$.

(2) If $A$ is a nonempty bounded closed subset of $X$ and $\operatorname{maxwp}(A)$ denotes the family of all $Z \in \mathscr{B}(X)$ for which the problem $\max (A, Z)$ is well posed, then $\mathscr{B}(X) \backslash \operatorname{maxwp}(A)$ is $\sigma$-porous in $\mathscr{B}(X)$.

The proof of assertion (1) uses in an essential way Stečkin's lens lemma and Proposition 2.11 .

Final remarks. I tried to give a general idea about some questions and results on the existence of nearest- and farthest-points for arbitrary subsets of Banach spaces, with emphasis on genericity and porosity results. Of course, a lot of interesting related questions were not included. Beside the results mentioned here, there are many other ones by De Blasi and Myjak, De Blasi and Zhivkov, De Blasi and Kenderov who, for a long period 
of time, systematically investigated various generic properties concerning nearest- and farthest-points problems. Reich and Zaslavski $[69,70,71,72]$ have recently also made contributions in this area. Penot $[63,64,65]$ obtained results on generic existence in optimization in connection with convex and nonsmooth analysis. There are also negative results, meaning the existence of closed convex and bounded antiproximinal sets in $\mathrm{Ba}$ nach spaces. A survey on this topic is given in [14] (see also [16]).

Another direction of investigation, very active lately, is that of the study of the socalled generalized optimization problems. One considers an absorbing convex subset $C$ of a normed space $X$ and the Minkowski functional

$$
p_{C}(x)=\inf \{\lambda>0: x \in \lambda C\}, \quad x \in X .
$$

The functional $p_{C}$ is subadditive and positive, but not necessarily absolutely homogeneous, since the set $C$ need not be symmetric. If $C$ is also radially bounded, then one calls $p_{C}$ an asymmetric norm on $X$. A study of analogs of the geometric properties of a Banach space in the case of a space with asymmetric norm was done by Zanco and Zucchi [82]. Contributions to existence results for optimization problems with respect to asymmetric norms have been made by De Blasi and Myjak [18], Li and Ni [53], Ni [62], and Maâden $[55,56]$.

\section{Acknowledgment}

I want to express my warmest thanks to the organizers of the International Workshop on Small Sets in Analysis which was held during June 25-30, 2003, at the Technion, Haifa, Israel, for their support.

\section{References}

[1] E. Asplund, Farthest points in reflexive locally uniformly rotund Banach spaces, Israel J. Math. 4 (1966), 213-216.

[2] - Fréchet differentiability of convex functions, Acta Math. 121 (1968), 31-47.

[3] V. S. Balaganskiu and L. P. Vlasov, The problem of the convexity of Chebyshev sets, Uspekhi Mat. Nauk 51 (1996), no. 6(312), 125-188 (Russian).

[4] P. Bandyopadhyay, The Mazur intersection property and farthest points, preprint, 1994.

[5] S. J. Bernau, On bare points, J. Aust. Math. Soc. 9 (1969), 25-28.

[6] P. A. Borodin, An example of a bounded approximatively compact set that is not compact, Uspekhi Mat. Nauk 49 (1994), no. 4(298), 157-158 (Russian), translated in Russian Math. Surveys 49 (1994), no. 4, 153-154.

[7] On convex approximatively compact sets and Efimov-Stechkin spaces, Vestnik Moskov. Univ. Ser. I Mat. Mekh. (1999), no. 4, 19-21, 71 (Russian), translated in Moscow Univ. Math. Bull. 54 (1999), no. 4, 18-20.

[8] J. M. Borwein, Some remarks on a paper of Ş. Cobzaş on antiproximal sets, Bull. Calcutta Math. Soc. 73 (1981), no. 1, 5-8.

[9] J. M. Borwein and S. Fitzpatrick, Existence of nearest points in Banach spaces, Canad. J. Math. 41 (1989), no. 4, 702-720.

[10] J. M. Borwein and R. C. O’Brien, Convex proximal sets with nonsurjective projections, preprint, 1978.

[11] R. D. Bourgin, Geometric Aspects of Convex Sets with the Radon-Nikodym Property, Lecture Notes in Mathematics, vol. 993, Springer, New York, 1983. 
[12] Ş. Cobzaş, Strongly nonproximinal sets in $C_{0}$, Rev. Anal. Numer. Teoria Aproximației 2 (1973), 137-141 (Romanian).

[13] _ Antiproximinal sets in some Banach spaces, Math. Balkanica 4 (1974), 79-82, papers presented at the Fifth Balkan Mathematical Congress (Belgrade, 1974).

[14] Antiproximinal sets in Banach spaces, Acta Univ. Carolin. Math. Phys. 40 (1999), no. 2, $43-52$.

[15]_, Generic existence of solutions for some perturbed optimization problems, J. Math. Anal. Appl. 243 (2000), no. 2, 344-356.

[16] - Antiproximinal sets in Banach spaces of continuous vector-valued functions, J. Math. Anal. Appl. 261 (2001), no. 2, 527-542.

[17] F. S. De Blasi and J. Myjak, On almost well-posed problems in the theory of best approximation, Bull. Math. Soc. Sci. Math. R. S. Roumanie (N.S.) 28(76) (1984), no. 2, 109-117.

[18] _ On a generalized best approximation problem, J. Approx. Theory 94 (1998), no. 1, 54 72.

[19] F. S. De Blasi, J. Myjak, and P. L. Papini, Porous sets in best approximation theory, J. London Math. Soc. (2) 44 (1991), no. 1, 135-142.

[20]_, On mutually nearest and mutually furthest points of sets in Banach spaces, J. Approx. Theory 70 (1992), no. 2, 142-155.

[21] R. Deville and V. E. Zizler, Farthest points in $w^{*}$-compact sets, Bull. Austral. Math. Soc. 38 (1988), no. 3, 433-439.

[22] J. Diestel and J. J. Uhl Jr., Vector Measures, Mathematical Surveys, no. 15, American Mathematical Society, Rhode Island, 1977.

[23] A. L. Dontchev and T. Zolezzi, Well-Posed Optimization Problems, Lecture Notes in Mathematics, vol. 1543, Springer, Berlin, 1993.

[24] M. Edelstein, Farthest points of sets in uniformly convex Banach spaces, Israel J. Math. 4 (1966), $171-176$.

[25] — Weakly proximinal sets, J. Approx. Theory 18 (1976), no. 1, 1-8.

[26] - Antiproximal sets, J. Approx. Theory 49 (1987), no. 3, 252-255.

[27] M. Edelstein and J. E. Lewis, On exposed and farthest points in normed linear spaces, J. Aust. Math. Soc. 12 (1971), 301-308.

[28] N. V. Efimov and S. B. Stečkin, Some properties of Čebyšev sets, Dokl. Akad. Nauk SSSR 118 (1958), 17-19 (Russian).

[29] _ Čebyšev sets in Banach spaces, Dokl. Akad. Nauk SSSR 121 (1958), 582-585 (Russian).

[30] - Support properties of sets in Banach spaces and Čebyšev sets, Dokl. Akad. Nauk SSSR 127 (1959), 254-257 (Russian).

[31] Approximative compactness and Chebyshev sets, Dokl. Akad. Nauk SSSR 140 (1961), 522-524 (Russian).

[32] K. Fan and I. Glicksberg, Some geometric properties of the spheres in a normed linear space, Duke Math. J. 25 (1958), 553-568.

[33] J. R. Giles, Convex Analysis with Application in the Differentiation of Convex Functions, Research Notes in Mathematics, vol. 58, Pitman, Massachusetts, 1982.

[34] G. Godini, A characterization of finite dimensional Banach spaces in the class of reflexive or separable Banach spaces, Rev. Roumaine Math. Pures Appl. 21 (1976), no. 7, 869-871.

[35] S. Hu and N. S. Papageorgiou, Handbook of Multivalued Analysis. Vol. I. Theory, Mathematics and Its Applications, vol. 419, Kluwer Academic, Dordrecht, 1997.

[36] D.-A. G. Kamuntavichyus, Category properties of a set of nonuniqueness points in the space $L_{\phi}$, Mat. Zametki 48 (1990), no. 3, 73-80, 158 (Russian).

[37] V. Kanellopoulos, On the convexity of the weakly compact Chebyshev sets in Banach spaces, Israel J. Math. 117 (2000), 61-69. 
[38] P. Kenderov, Points of single-valuedness of multivalued metric projections, C. R. Acad. Bulgare Sci. 29 (1976), no. 6, 773-775.

[39] - Points of continuity of semi-continuous multivalued mappings and application to the theory of metric projections, Mathematics and education in mathematics, Proc. 4th Spring Conf., Bulgarian Mathematical Society, Sofia, 1978, pp. 191-197.

[40] _ Dense strong continuity of pointwise continuous mappings, Pacific J. Math. 89 (1980), no. $1,111-130$.

[41] S. V. Konyagin, Approximation properties of arbitrary sets in Banach spaces, Dokl. Akad. Nauk SSSR 239 (1978), no. 2, 261-264 (Russian).

[42] - Approximation properties of closed sets in Banach spaces and the characterization of strongly convex spaces, Dokl. Akad. Nauk SSSR 251 (1980), no. 2, 276-280 (Russian).

[43] Connectedness of sets in problems of best approximation, Dokl. Akad. Nauk SSSR 261 (1981), no. 1, 20-23 (Russian).

[44] - Sets of points of nonemptiness and continuity of a metric projection, Mat. Zametki 33 (1983), no. 5, 641-655 (Russian).

[45] _ Sets of existence of elements of best approximation, Theory of Functions and Approximations, Part 2 (Saratov, 1984), Saratov. Gos. Univ., Saratov, 1986, pp. 136-139 (Russian).

[46] Letter: "On points of existence of elements of best approximation by sets in reflexive spaces" [East J. Approx. 1 (1995), no. 1, 141-142], East J. Approx. 1 (1995), no. 4, 576.

[47] On points of existence of elements of best approximation by sets in reflexive spaces, East J. Approx. 1 (1995), no. 1, 141-142.

[48] On approximation properties of arbitrary closed sets in Banach spaces, Fundam. Prikl. Mat. 3 (1997), no. 4, 979-989 (Russian).

[49] S. V. Konyagin and I. G. Tsar'kov, Efimov-Stechkin spaces, Vestnik Moskov. Univ. Ser. I Mat. Mekh. (1986), no. 5, 20-27, 99 (Russian).

[50] K. S. Lau, Farthest points in weakly compact sets, Israel J. Math. 22 (1975), no. 2, 168-174.

[51] Almost Chebyshev subsets in reflexive Banach spaces, Indiana Univ. Math. J. 27 (1978), no. $5,791-795$.

[52] C. Li, On mutually nearest and mutually furthest points in reflexive Banach spaces, J. Approx. Theory 103 (2000), no. 1, 1-17.

[53] C. Li and R. Ni, Derivatives of generalized distance functions and existence of generalized nearest points, J. Approx. Theory 115 (2002), no. 1, 44-55.

[54] C. Li and H.-K. Xu, Porosity of mutually nearest and mutually furthest points in Banach spaces, J. Approx. Theory 125 (2003), no. 1, 10-25.

[55] A. Maâden, C-nearest points and the drop property, Collect. Math. 46 (1995), no. 3, 289-301.

[56] On the C-farthest points, Extracta Math. 16 (2001), no. 2, 211-222.

[57] S. Mazur, Über schwache Konvergenz in den Räumen (L $\left.L^{p}\right)$, Studia Math. 4 (1933), 128-133 (German).

[58] R. E. Megginson, An Introduction to Banach Space Theory, Graduate Texts in Mathematics, vol. 183, Springer, New York, 1998.

[59] T. D. Narang, A study of farthest points, Nieuw Arch. Wisk. (3) 25 (1977), no. 1, 54-79.

[60] Convexity of Chebyshev sets, Nieuw Arch. Wisk. (3) 25 (1977), no. 3, 377-402.

[61] Uniquely remotal sets are singletons, Nieuw Arch. Wisk. (4) 9 (1991), no. 1, 1-12.

[62] R. Ni, Existence of generalized nearest points, Taiwanese J. Math. 7 (2003), no. 1, 115-128.

[63] J.-P. Penot, Proximal mappings, J. Approx. Theory 94 (1998), no. 2, 203-221.

[64] Well-behavior, well-posedness and nonsmooth analysis, Pliska Stud. Math. Bulgar. 12 (1998), 141-190.

[65] _ Genericity of well-posedness, perturbations and smooth variational principles, SetValued Anal. 9 (2001), no. 1-2, 131-157. 
[66] R. R. Phelps, A representation theorem for bounded convex sets, Proc. Amer. Math. Soc. 11 (1960), 976-983.

[67] Convex Functions, Monotone Operators and Differentiability, 2nd ed., Lecture Notes in Mathematics, vol. 1364, Springer, Berlin, 1993.

[68] S. Reich, Approximate selections, best approximations, fixed points, and invariant sets, J. Math. Anal. Appl. 62 (1978), no. 1, 104-113.

[69] S. Reich and A. J. Zaslavski, Well-posedness and porosity in best approximation problems, Topol. Methods Nonlinear Anal. 18 (2001), no. 2, 395-408.

[70] Well-posedness of generalized best approximation problems, Nonlinear Funct. Anal. Appl. 7 (2002), no. 1, 115-128.

[71] - A porosity result in best approximation theory, J. Nonlinear Convex Anal. 4 (2003), no. $1,165-173$.

[72] - Best approximations and porous sets, Comment. Math. Univ. Carolin. 44 (2003), no. 4, 681-689.

[73] B. Ricceri, A general mutiplicity theorem for certain nonlinear equations in Hilbert space, to appear in Proc. Amer. Math. Soc.

[74] I. Singer, Some remarks on approximative compactness, Rev. Roumaine Math. Pures Appl. 9 (1964), 167-177.

[75] V. I. Šmulian, Sur la dérivabilité de la norme dans l'espace de Banach, C. R. (Doklady) Acad. Sci. URSS (N. S.) 27 (1940), 643-648 (French).

[76] S. B. Stečkin, Approximation properties of sets in normed linear spaces, Rev. Math. Pures Appl. 8 (1963), 5-18 (Russian).

[77] S. Straszewicz, Über exponierte Punkte abgeschlossener Punktmengen, Fund. Math. 24 (1935), 139-143 (German).

[78] L. P. Vlasov, Approximative properties of sets in normed linear spaces, Uspehi Mat. Nauk 28 (1973), no. 6(174), 3-66 (Russian).

[79] L. Zajíček, On the points of multivaluedness of metric projections in separable Banach spaces, Comment. Math. Univ. Carolin. 19 (1978), no. 3, 513-523.

[80] - Porosity and $\sigma$-porosity, Real Anal. Exchange 13 (1987/1988), no. 2, 314-350.

[81] _ On $\sigma$-porous sets in abastract spaces, preprint, 2004.

[82] C. Zanco and A. Zucchi, Moduli of rotundity and smoothness for convex bodies, Boll. Un. Mat. Ital. B (7) 7 (1993), no. 4, 833-855.

[83] N. V. Zhivkov, Generic Gâteaux differentiability of locally Lipschitzian functions, Constructive Function Theory '81 (Varna, 1981), Publ. House Bulgar. Acad. Sci., Sofia, 1983, pp. 590594.

[84] _ Generic Gâteaux differentiability of directionally differentiable mappings, Rev. Roumaine Math. Pures Appl. 32 (1987), no. 2, 179-188.

Stefan Cobzaş: Faculty of Mathematics and Computer Science, Babeş-Bolyai University, 3400 ClujNapoca, Romania

E-mail address: scobzas@math.ubbcluj.ro 


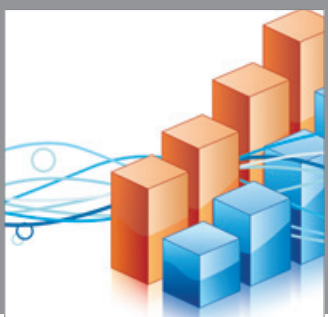

Advances in

Operations Research

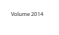

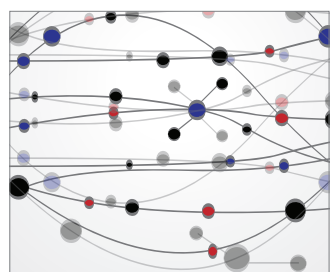

\section{The Scientific} World Journal
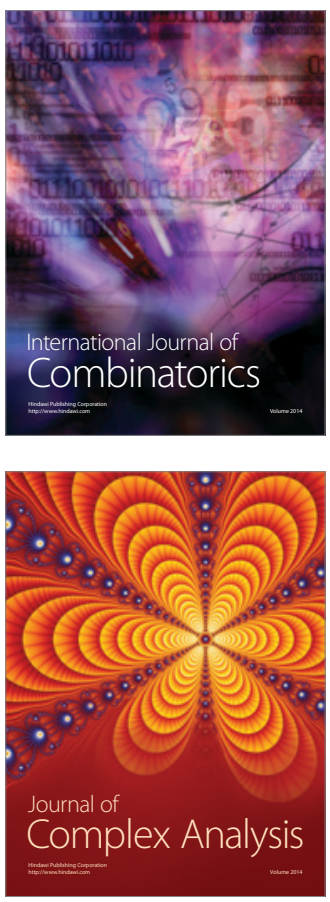

International Journal of

Mathematics and

Mathematical

Sciences
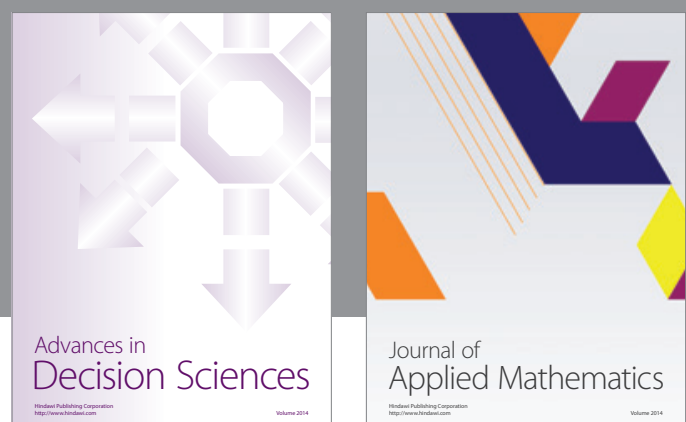

Journal of

Applied Mathematics
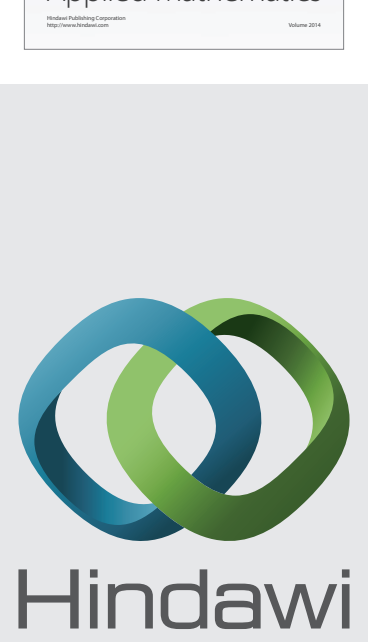

Submit your manuscripts at http://www.hindawi.com
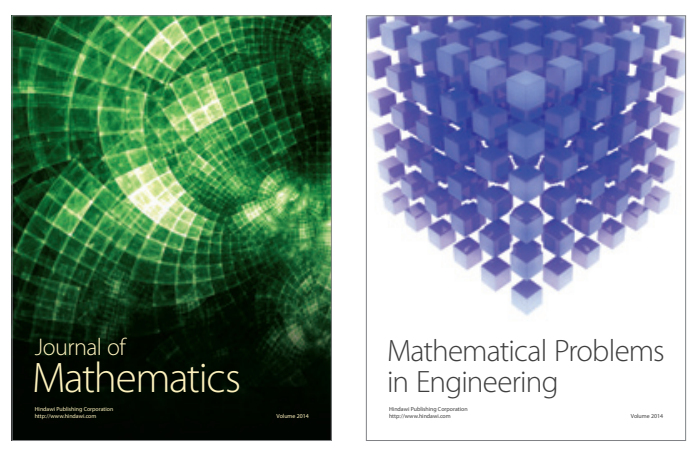

Mathematical Problems in Engineering
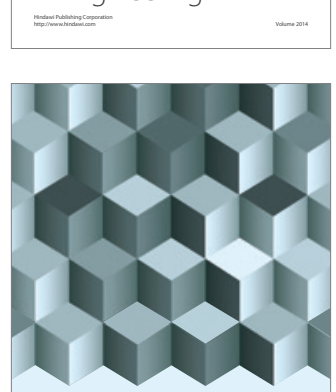

Journal of

Function Spaces
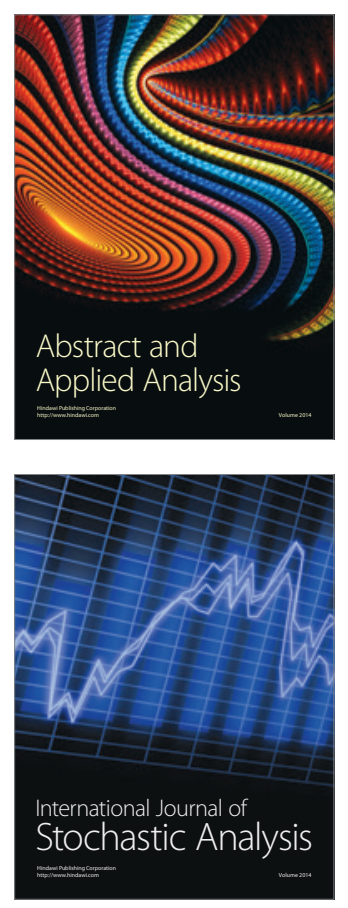

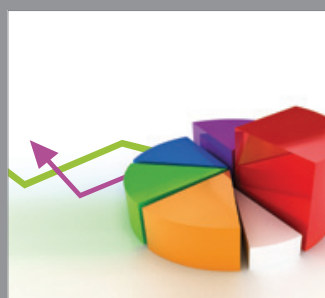

ournal of

Probability and Statistics

Promensencen
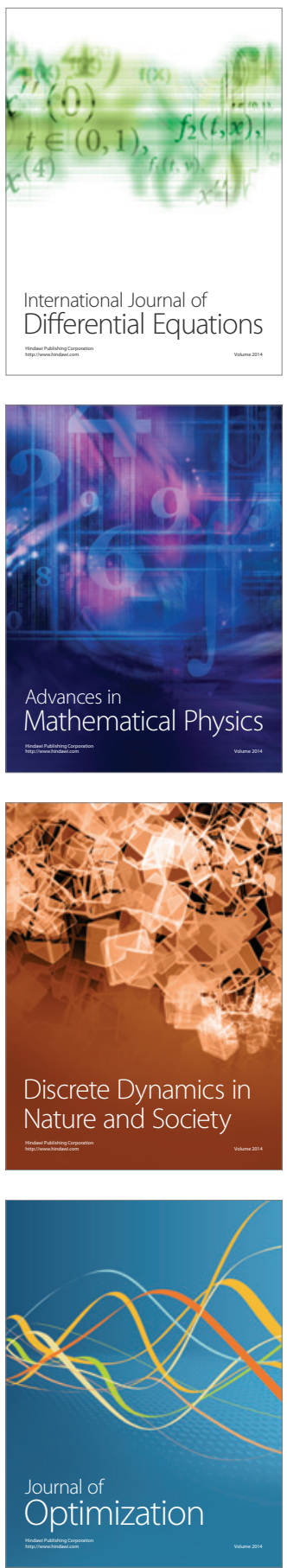